\title{
An experimental study of wave run-up at a steep beach
}

\author{
Submitted to Journal of Fluid Mechanics \\ Atle Jensen, Geir K. Pedersen \& Deborah J. Wood \\ Mechanics Division, Department of Mathematics, \\ University of Oslo
}

This paper focus on experiments on run-up of strongly nonlinear waves on a moderately steep beach of $10.5^{\circ}$ inclination. Velocity fields are obtained by the PIV (Particle Image Velocimetry) technique. Acceleration measurements are also attempted, but it is difficult to obtain useful results in every case. In addition free surface profiles are extracted from digital images and wave resistance probes.

Several non-breaking incident waves are measured and compared with theory in deep water as well as under run-up. However, the main theme is run-up of steep waves at the brink of breaking, that is assumed to be a good starting point approaching a detailed study of bore runup. In some cases these are found to be temporarily slightly overturning in the first stages of run-up, without developing neither a plunger nor appreciable spilling. This feature is discussed in view of acceleration measurements and with reference to the dam-break problem. Effects of scaling, as well as three dimensional features observed for the steepest waves, are also briefly discussed.

\section{Introduction}

The present study is particularly motivated by studies of tsunamis due to earthquakes and submarine slides. Since 1990 we have not seen any event comparable to the larger historic ones. Still, thousands of lives have been lost in tsunami disasters, in addition to huge damage to coastal installations and buildings. A complete study of the tsunami phenomenon involves a series of subtopics of fundamental importance both from a geological and hydrodynamic point of view. However, the ultimate goal is always prediction of the tsunami impact and run-up on shores, breakwaters and harbor installations. Run-up of waves has been an important theme in hydrodynamic wave theory for many decades. In addition to tsunamis the topic concerns a number of other applications, for instance coastal erosion and sedimentation by swells, construction of absorbing beaches in laboratory wave tanks and coastal disintegration of internal waves on the pycnocline. In this paper we will examine the flow structure of surface waves running up a beach by PIV (Particle Image Velocimetry) measurements as well as numerical models. Particular emphasis is put on the early crucial stages of the run-up.

Theoretical treatment of run-up has traditionally been based on nonlinear shallow water theory. On plane slopes some analytical solutions have been found for both 
non-breaking waves, Carrier \& Greenspan (1958), and bores Keller et al. (1960); Shen \& Meyer (1963). In numerical computations on an Eulerian grid, points must be dynamically included or exempted from the computational (wetted) domain according to the motion of the shoreline, as reported by, for instance, Hibberd \& Peregrine (1979), Flather \& Heaps (1975) and Kowalik \& Murty (1993). In such methods crude bore models may be included as jumps, that are sustained by some scheme for energy extraction and sometimes sharpened by particular correction procedures. Naturally, no details of the bore front are appropriately described, while the global characteristics may be reproduced well. An alternative is the use of Lagrangian coordinates as is found in Pedersen \& Gjevik (1983), Zelt \& Raichlen (1990), Johnsgard \& Pedersen (1997) and Johnsgard (1999). While Lagrangian coordinates yields a simpler and more accurate description of the shoreline in simpler cases, large scale run-up in complex geometries may produce strongly deformed grid that imply regular re-meshing. More general run-up models are reported by Lin et al. (1999), Grilli \& Svendsen (1990) and others.

Quite a number of experimental investigations on run-up have been reported. Traditionally surface elevations have been measured by wave gauges or the maximum run-up height has been observed visually. Some of the older work is surveyed in Meyer \& Taylor (1972), while more recent results for solitons are given in Synolakis (1987).

The measurement of velocities within a wave is more complicated. However, in the last decades several methods have been developed that have accomplished this with increasing success as the technology improves. Nadoaka et al. (1989) used fibreoptic Laser-Doppler Anemometer (LDA) to study the turbulent flow field under a breaking wave. They considered a slope of 1 in 20 , and found that under breaking waves there is a particular type of large scale eddy structure. Many photographs and analysis of Reynolds stress give insight into the type of structure of the eddies. Ting \& Kirby (1995) and Ting \& Kirby (1996) looked at a strong plunging and a spilling breaker respectively. The slope in these studies was more gentle, 1 in 35 . Both water surface elevations and water particle velocities were measured using a wave gauge and a fibre-optic LDA respectively. The experiments had to be run twice to obtain the velocity components in the vertical and horizontal directions. These studies were purely experimental, however they gave good descriptions of the structure of the turbulence in both types of wave. They studied turbulence and dissipation in the two types of waves finding, among other things, that dissipation of turbulent kinetic energy is much faster for a plunging breaker.

Using the newer method particle image velocimetry (PIV) the full velocity field can be obtained. PIV can still not be used in aerated regions, but, applied with care, the method can be successful in capturing velocities in some other difficult situations such as in a crest of a broken wave (see for example Craig \& Thieke (1996)).

Lin et al. (1999) used the volume of fluid model (VOF) described in Lin \& Liu $(1998 a, b)$ to examine solitary waves running up and down a beach. They compared the computations with PIV experiments for a rather long non-breaking wave on a steep slope of $30^{\circ}$, and found good agreement. Breaking waves on a much milder slope $\left(2.88^{\circ}\right)$ were also computed, but were compared only to the surface measurements of Synolakis (1987); again with good agreement.

Chang \& Liu (1999) is an experimental study using PIV to investigate turbulence in a spilling breaker. They examined the number of experiments required for obtaining 
good representations of averaged quantities and concluded that 16 seemed appropriate. They also looked at the generation and decay of turbulence. The 'roller' production theory was thought to be reasonable, and that a quasi-steady state was reached after the passage of about five breaking waves.

In addition to the study of velocities and turbulence in waves there are some studies on the measurement of accelerations. The measurement of accelerations is difficult because it involves the subtraction of two velocity fields, both of which are subject to noise. In addition, to obtain physical meaning from the accelerations the time difference between the two velocity fields must be small. The difference in the velocity fields is then divided by the time interval, a small quantity, causing more error. Chang \& Liu (1998) is a very brief paper, but does give some estimates of accelerations in the overturning jet. Jakobsen et al. (1997) describe the techniques for measuring accelerations with PIV and give some analysis on the errors which occur using this technique. They claim to be able to measure accelerations to within $3-7 \%$. Jensen et al. (2001) use a two camera system for measuring accelerations in Stokes waves. The measurements of accelerations in short crested waves gave the best results with relative standard deviation down to $2 \%$.

While surf zone dynamics has been the topic for a series of papers there has been less work on the detailed velocity distributions for a wave running up a beach. Most tsunamis and swells on gentle slopes break and are turned into bores as they approach the shore. However, close to the very shoreline the breaker collapses and vanish and a thin, smooth, though turbulent, wedge of water shoots up the beach. Due to the elongated shape of this tongue of liquid, gravity will often dominate over horizontal pressure gradients. A crude conception of the run-up itself may then be a set of independent fluid particles following parabolic trajectories in time, as long as frictional effects are neglected (see Shen \& Meyer (1963)). Thus, the most crucial step for determination of the run-up is the maximum velocities produced in the bore collapse. Since this process may be influenced by details in the bore structure, as well as the width compared to amplitude, it is not likely that it is properly described by any of the simpler bore models in shallow water theory. In fact, the run-up of steep non-breaking waves follows a similar pattern: the wave front becomes steeper until the shore is reached, then a rapid transition to a thin jet occurs. As for bores, the run-up tip then is closely approximated by a parabolic function of time (see figure 22). Therefore, the study of nearly breaking waves may be a good starting point for revealing the important mechanisms in the bore collapse. Moreover, when distinct breaking does not occur we also avoid aeration and strong turbulence production that will cause severe problems both for the PIV measurements and the modeling. Run-up of nearly breaking waves, of appreciable amplitudes, is most easily obtained on moderately steep slopes. This also enables short total propagation distances that enhance repeatability, which is essential. In particular the PIV field of view (FOV) is relatively small, $12 \mathrm{~cm}$ by $12 \mathrm{~cm}$ say, implying that the different stages of the run-up must be examined with different FOVs and runs of the experiment. PIV measurements are also sensitive to seeding of particles. So it is important that repeatability can be used to identify "stray" results. We will compare measurements with a Boussinesq model, as well as soliton solutions based on full potential theory. The main intention behind the comparison with theory is primarily to check the measurements, while some emphasis is also put on the applicalibility of the Boussinesq model. However, we plan to pursue these 
and other computational aspects further in a forthcoming paper, employing also more general models.

\section{Experimental setup}

\subsection{Measurement techniques}

Particle Image Velocimetry (PIV) were used for velocity measurements. A light sheet was created by a continuous wave $(\mathrm{CW})$ laser and a scanning beam system. The CW beam was modulated with an acousto-optic modulator which selects two or more pulses for PIV recordings. High-sensitive CCD cameras with a resolution of $1280 \times$ 1024pixels and 12-bit digital outputs were used for image acquisition. The start of capturing images was triggered by the same computer which controls the wave maker. The triggering has a variability of less than the scanning period of the PIV-system, set between 1 to 12 microseconds in the experiments. This will slightly affect the repeatability. In all experiments an interrogation window of $64 \times 64$ pixels with an overlap of $50 \%$ was used. The post-processing procedure and the PIV system are described in more detail by Jensen et al. (2001). Furthermore, conifer pollen was added to the flow as seeding particles. The presence of the organic pollen led to growth of bacteria and algae that increased the opacity of the water. As a result the water had to be changed regularly and new particles added. Hence, in the last few measurements in deep water we switched to $50 \mu \mathrm{m}$ polyamid particles. No significant differences between the polyamid and the reported pollen measurement were observed.

The optimal camera angle is perpendicular to the light sheet. Much of the measurements are made in fluid regions above the equilibrium water level and is calibrated according to the sheet being viewed through water (until the side wall of the tank). Then, for instance, when the water level is low the surface may be viewed nearly horizontally, or even slightly from above. Consequently, the neighborhood of the surface will be distorted due to refraction and reflection effects at the free surface. Hence, in some images there was some loss of information near the free surface of the fluid. Naturally, refraction effects at the tank sidewall will lead to nonlinearities in the mapping between physical coordinates and the image frame that are not taken into account by the PIV software. However, these errors were small.

In addition to the velocity measurements by PIV, wave heights were measured by resistance wave probes at two selected locations. The digital images from the PIV system were also used to estimate the free surface location with a bitmap viewer. Also the digitized surfaces are sometimes affected by optical effects. In addition the accuracy of the surface image itself is reduced due to the high exposure time needed to capture the small pollen particles.

\subsection{Wave tank and generation}

All the reported experiments were performed in a wave tank at the Hydrodynamic Laboratory, Department of Mathematics, University of Oslo. The wave tank is $1 \mathrm{~m}$ high, $0.5 \mathrm{~m}$ wide and $25 \mathrm{~m}$ long, even though only a section of $6 \mathrm{~m}$ was used in the present experiments. The tank is constructed with deviations in the depth and width 


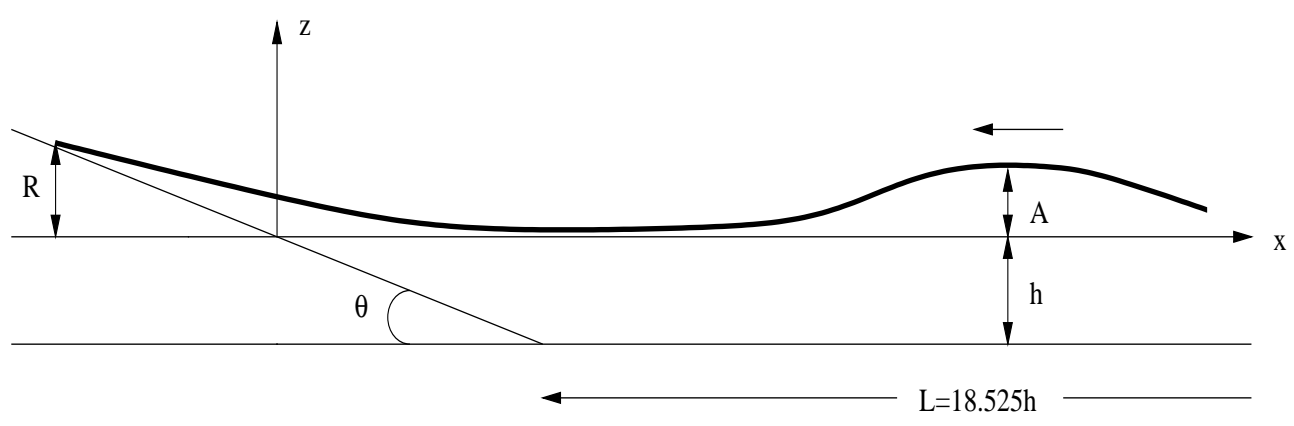

Figure 1: Definition sketch of the wave tank.

less than $0.5 \mathrm{~mm}$. At one end of the tank waves were generated by a hydraulic piston attached to a vertical paddle.

A definition sketch of the wave tank and the beach is in figure 1. To allow for large amplitudes and run-up height $(R)$ most of the experiments were carried out with an equilibrium depth $(h)$ equal to $20 \mathrm{~cm}$. A selection of the experiments were scaled down and performed in $10 \mathrm{~cm}$ depth, to get an indication of the scaling effects. In all run-up experiments a rather steep beach, with an inclination $\theta=10.54^{\circ}$, at a distance $L=18.525 h$ from the undisturbed position of the wave maker. The beach is made of perspex that expand slightly due to water absorption and buckle. Even though the resulting deformation of beach is small (less than $1 \mathrm{~mm}$ ), it may have implications for three dimensional features reported subsequently.

With regard to tsunami applications the most appropriate choice would be incident waves with a long elevation following a steep front, or a long trough terminated by a steep gradient, in either case reflecting possible characteristics of tsunamis in coastal waters. However, limitations on the wave paddle motion rendered the creation of such waves with appreciable amplitudes impossible. Instead a small selection of shorter waves were made. In principle the paddle position is governed explicitly by an input voltage, provided by a computer, yielding approximately $4 \mathrm{~cm}$ displacement per volt. However, whilst this is correct for the final paddle location, a substantial but repeatable, delay of the paddle response may be observed in cases with large paddle accelerations. For the experiments in $20 \mathrm{~cm}$ depth we employed the voltage distribution, involving the parameters $T$ and $V_{\max }$

$$
V(t)=30 V_{\max } T\left(\frac{1}{5}\left(\frac{t}{T}\right)^{5}-\frac{1}{2}\left(\frac{t}{T}\right)^{3}+\frac{1}{3}\left(\frac{t}{T}\right)^{2}\right),
$$

for $t<T$. Subsequently, the paddle was still for a while, before being restored to its original position. In every experiment the actual paddle position is measured, by means of a $1 \mathrm{kHz}$ magnetic digital measurement system. We have focused on four waves that are associated with the parameters showed in the table in figure 2 .

Case $I$ is a long wave, but not a soliton when it propagates in deep water. This wave should be an obvious candidate for applications of long wave theory. The next wave (II) is steep and breaks during generation, but there is no sign of breaking during run-up. The intermediate wave (III) is meant to be challenging for long wave theory. Case IV is selected to be a wave on the brink of breaking in the steepest phase during 

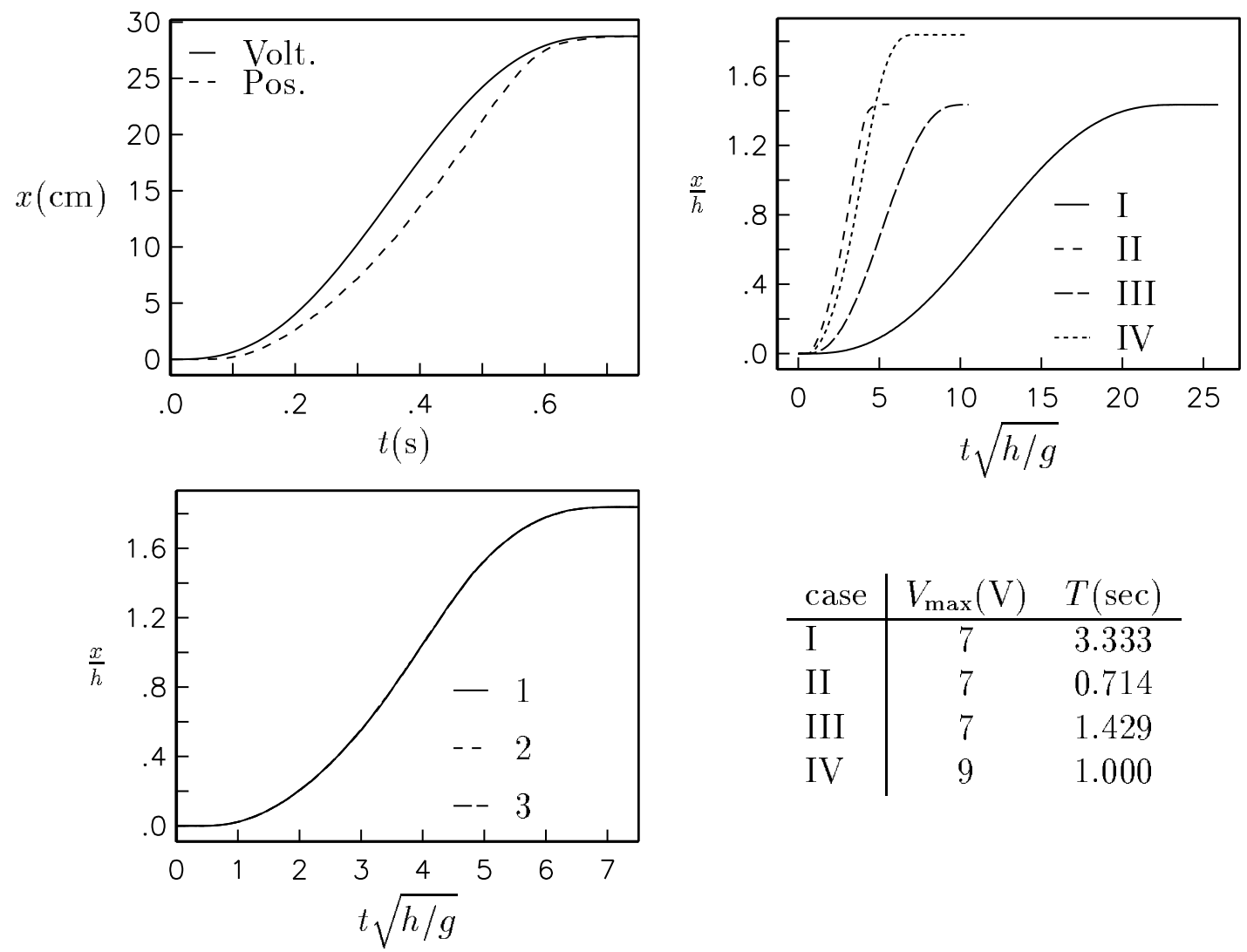

\begin{tabular}{l|cc} 
case & $V_{\max }(\mathrm{V})$ & $T(\mathrm{sec})$ \\
\hline I & 7 & 3.333 \\
II & 7 & 0.714 \\
III & 7 & 1.429 \\
IV & 9 & 1.000
\end{tabular}

Figure 2: Upper left panel: renormalized input voltage as compared to the paddle motion for case II. Upper right: Normalized paddle motions for all cases. Lower left: Repeatability of paddle motion. Different runs corresponding to case $I V$ are displayed. Lower right: paddle parameters.

run-up, while no breaking or spilling is observed until this stage (see figure 9 and 15). In the top diagram of figure 2 we have compared the normalized voltage distribution to displacement for case II. Paddle displacements for all cases and paddle displacements for different runs of case IV to check the repeatability are shown in the middle and bottom diagrams respectively.

With a smaller depth, $h=10 \mathrm{~cm}$, we want to reproduce the normalized paddle motions from figure 2. Save for viscous and capillary effects, we should then obtain a wave motion similar to the one for $h=20 \mathrm{~cm}$, with all lengths halved and the time scale reduced by a factor $\sqrt{\frac{1}{2}}$. Due to the differences in hydrodynamic resistance, as compared to other types of resistance in the wave maker system, this is not obtained simply by scaling the voltage distribution. Instead the correct voltages for $h=10 \mathrm{~cm}$ are obtained by an iteration procedure where deviations of the measured paddle motion from the desired one, is used to adjust the input voltage. Naturally, any kind of paddle motion could be obtained in a similar manner.

\section{Incident waves; constant depth}

To characterize incident waves and verify the PIV procedure a set of PIV and wave gauge measurements were taken in deep water, either in the absence of or at the front 


\begin{tabular}{l||c|c|c|c} 
Case & $\frac{A}{h}$ & $t_{t r}$ & $\Delta t_{1}$ & Field of view \\
\hline \hline I & 0.12 & $3.85 \mathrm{~s}$ & $12 \mathrm{mS}$ & $-18.1<x<7.7 \mathrm{~cm},-11.5<y<9 \mathrm{~cm}$ \\
II & 0.53 & $2.57 \mathrm{~s}$ & $6 \mathrm{mS}$ & $-17.7<x<8.1 \mathrm{~cm},-3.7<y<17 \mathrm{~cm}$ \\
III & 0.335 & $2.97 \mathrm{~s}$ & $10 \mathrm{mS}$ & same as I \\
IV & 0.655 & $2.57 \mathrm{~s}$ & $4 \mathrm{mS}$ & same as II
\end{tabular}

Table 1: The four cases in $20 \mathrm{~cm}$ depth. $A$ is the wave height, $h$ is the water depth and $t_{t r}$ is the trigger time of the PIV measurements. $\Delta t_{1}$ is the time between the two acquired images which are used in the calculation of the full velocity field.

of the beach. In either case, reflection from the slope, beach or end wall did not affect the leading crest of the wave markedly.

Comparisons of measured velocities and wave heights are made with solitary waves as obtained by the method of Tanaka (1986) as well as a modified version of the Boussinesq model from Pedersen \& Gjevik (1983) (see appendix B).

Time gauge measurements, without a beach and $3.775 \mathrm{~m}$ from the paddle, were performed for all cases with $h=20 \mathrm{~cm}$ as the depth. The comparison of these measurements with theory is shown in figure 3. Case II through IV are close to the soliton solution of Tanaka. The long and moderately nonlinear wave of case I has not yet produced a soliton at this stage, but is, on the other hand, well described by the Boussinesq model. For case II and IV the Boussinesq model deviates strongly from measurements, while the deviations are smaller, but still appreciable, for case III. It is noteworthy that the digitized surfaces from images (table 2) yield a slightly lower amplitude for case III, thereby indicating a somewhat reduced error for the Boussinesq model.

In all the PIV measurements the distance from the wave maker to the local origin of the FOV is $3.705 \mathrm{~m}$. Other crucial parameters for the PIV measurements in deep water are listed in table 1 . Surfaces from the images yield slightly different results from the wave gauges. Measured and computed amplitudes are summarized in table 2 .

Figure 4 contains the full velocity field (case IV), both for PIV and the Tanaka soliton solution. The interface is thick due to the exposure time of the acquisition. Still, we observe good agreement for the surface, while experiments and theory are almost identical concerning velocities. In figure 5 surface profiles from the PIV images (cases III and IV) are compared to theory. The soliton solution of Tanaka agree well with experiments for both cases. As would be expected the Boussinesq solution is considerably better for case III.

Vertical cross sections of the velocity fields are displayed in figure 6. Comparing measurements and theory we find the same pattern as for surfaces: good comparison with the Boussinesq solution for case I and III and good agreement with the Tanaka solution for case II through IV.

In order to verify the repeatability the standard deviation is calculated (figure 7) from five different runs. It is largest beneath the free surface. This is to be expected because the noise in the correlation peak increases when the particles cross-correlate with the highly exposed surface. By a combination of PIV and the reflective mode of the free-surface gradient detector (FSGD) technique it is possible to compute velocity vectors somewhat closer to the surface, see Dabiri \& Gharib (2001), but this is not attempted herein. 
Case I

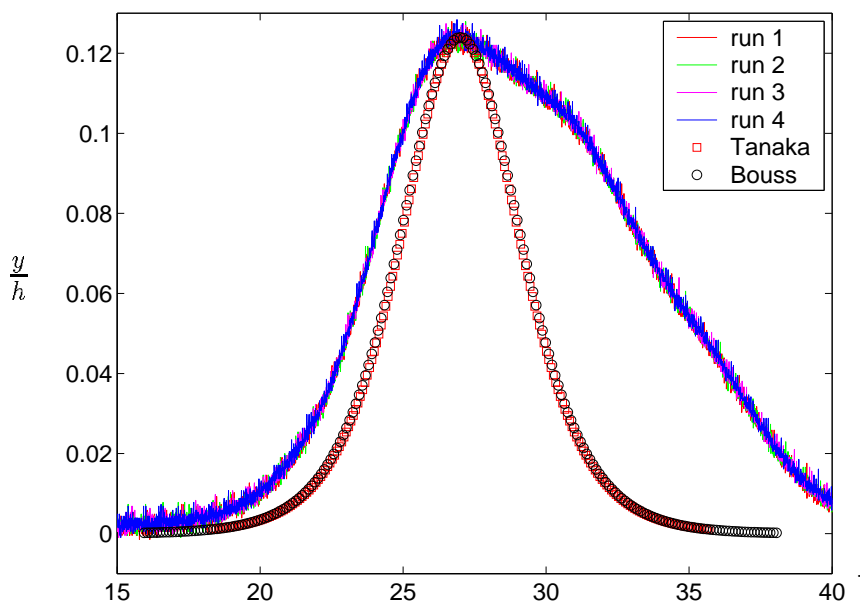

Case III

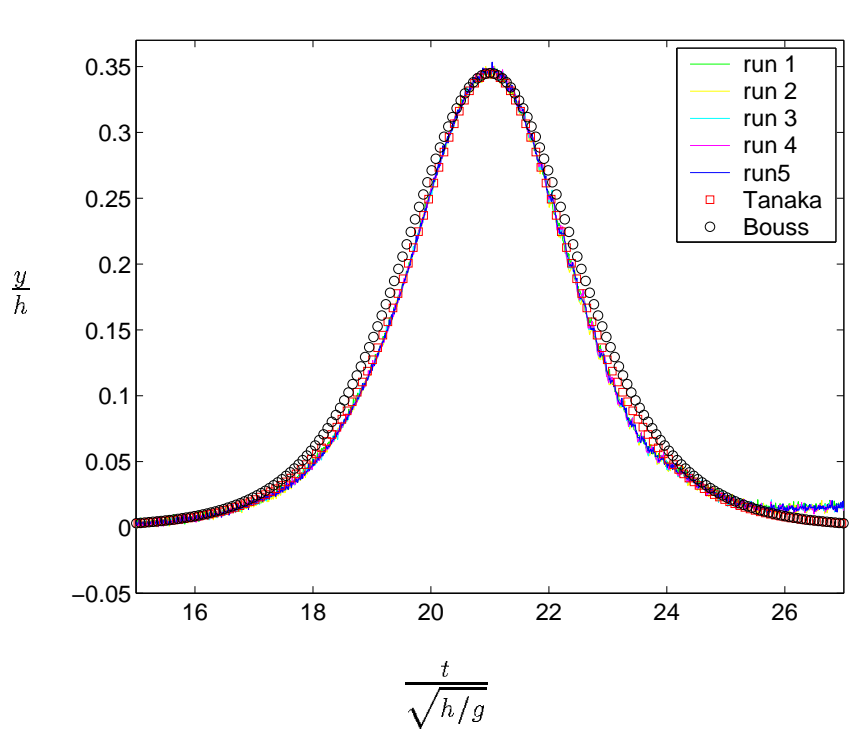

Case II

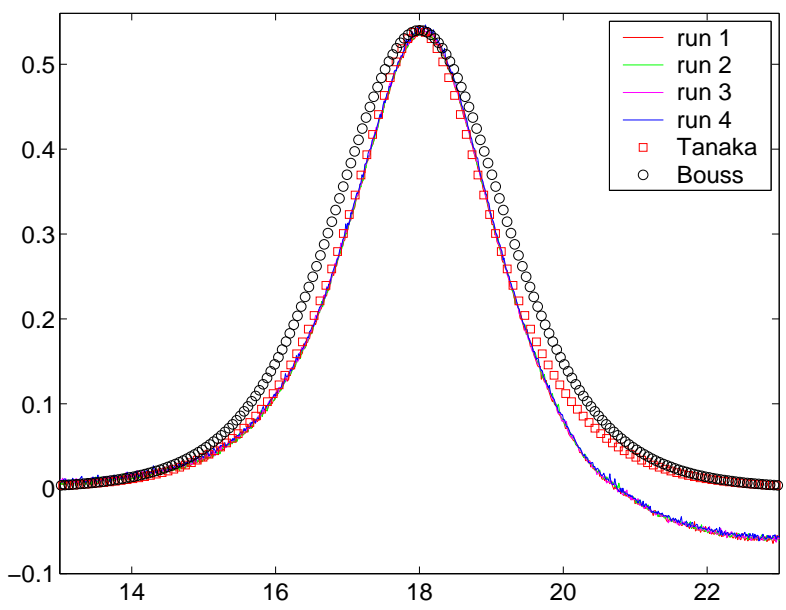

Case IV

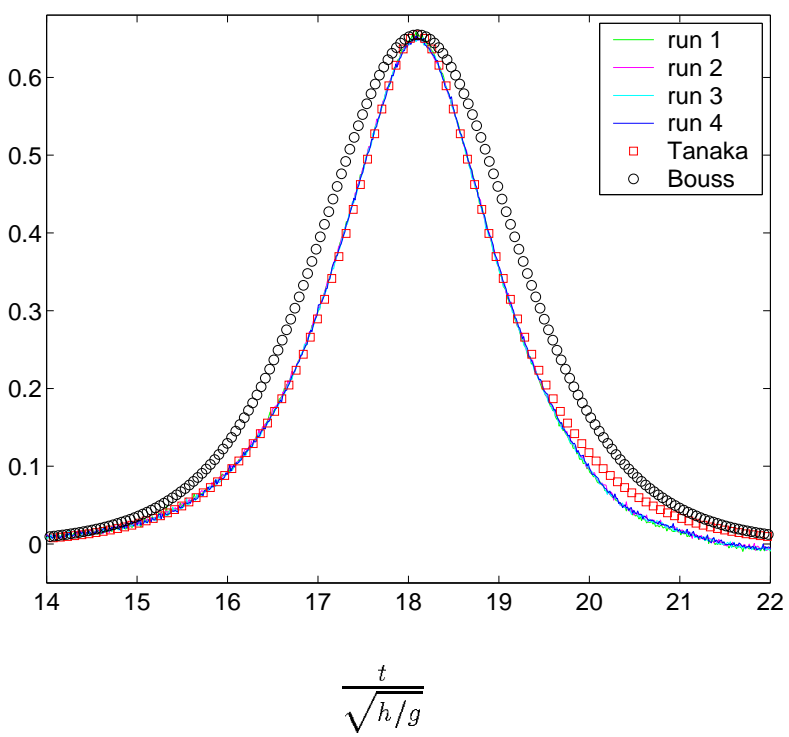

Figure 3: Wave elevation in deep water.

\begin{tabular}{r||r|r|r|r} 
case & $A(\mathrm{PIV})$ & $A$ (Bouss.) & $\Delta x$ (Bouss.) & $A(\mathrm{WG})$ \\
\hline \hline IV & 13.25 & 11.18 & -9.1 & 13.2 \\
III & 6.64 & 6.54 & -3.2 & 6.9 \\
II & 10.73 & 9.12 & -9.5 & 10.7 \\
I & 2.4 & 2.53 & - & 2.5
\end{tabular}

Table 2: Amplitudes $A$ and in $\mathrm{cm}$, from PIV images (PIV), Boussinesq simulations (Bouss.) and wave gauges (WG). The phase lag, $\Delta x$ is defined as the difference (in $\mathrm{cm}$ ) between the maximum of digitized surfaces and the Boussinesq solution at $t=t_{t r}$. Negative values correspond to delayed simulated waves. The digitized surface of case $I$ is hampered with error due to unfavourable camera angle. Hence, the extracted amplitude is uncertain to a few $\mathrm{mm}$. 


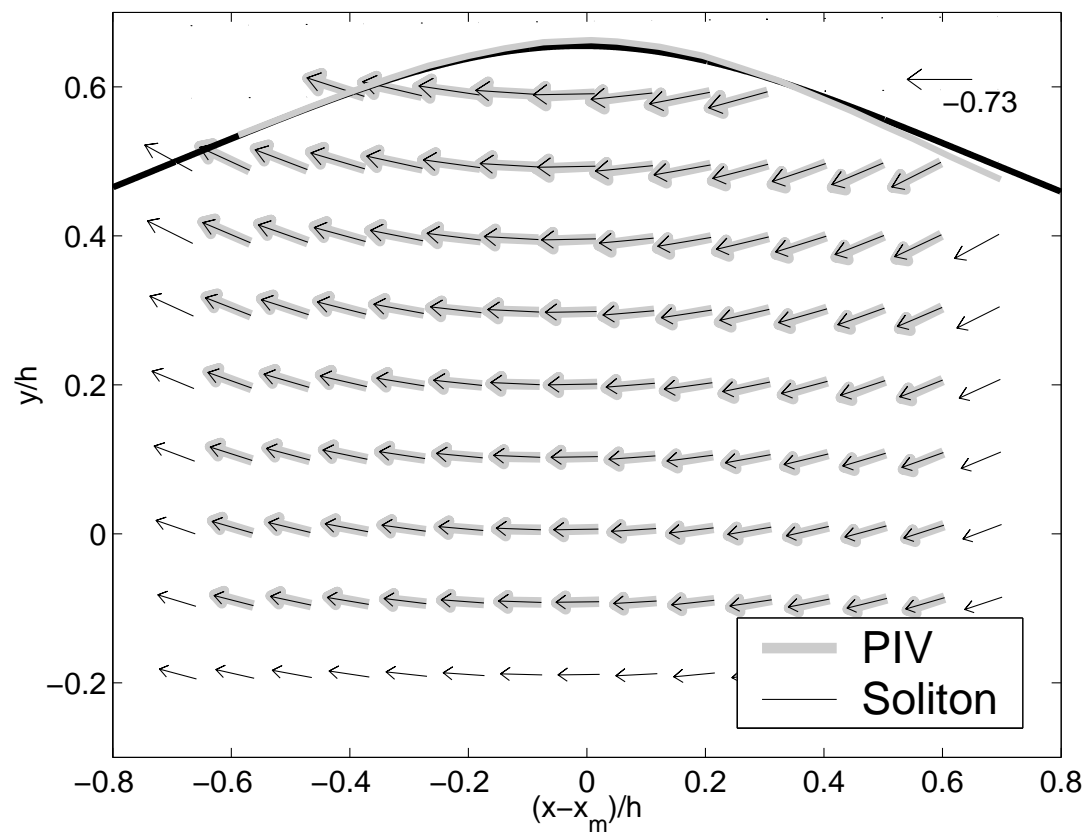

Figure 4: Full velocity field with exctracted surfaces, both experiments and theory. Grey is PIV, black is Tanaka (soliton) and $x_{m}=5.3 \mathrm{~cm}$ or $3.758 \mathrm{~m}$ from the paddle.
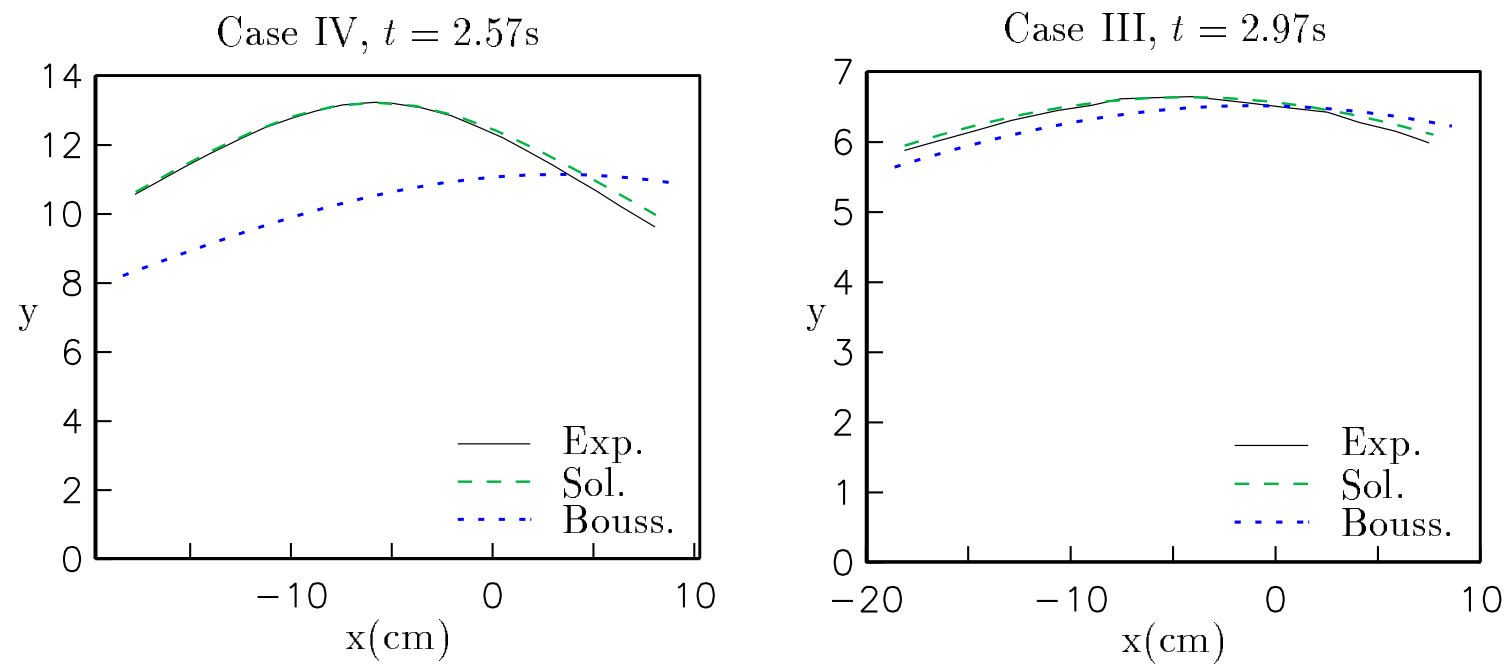

Figure 5: Surfaces in deep water of case III and IV. Experimental values (Exp.) are compared to data from the Boussinesq solver (Bouss.) and the soliton solution of Tanaka (Sol.). The origin of the coordinate systems is $3.705 \mathrm{~m}$ from the paddle. 

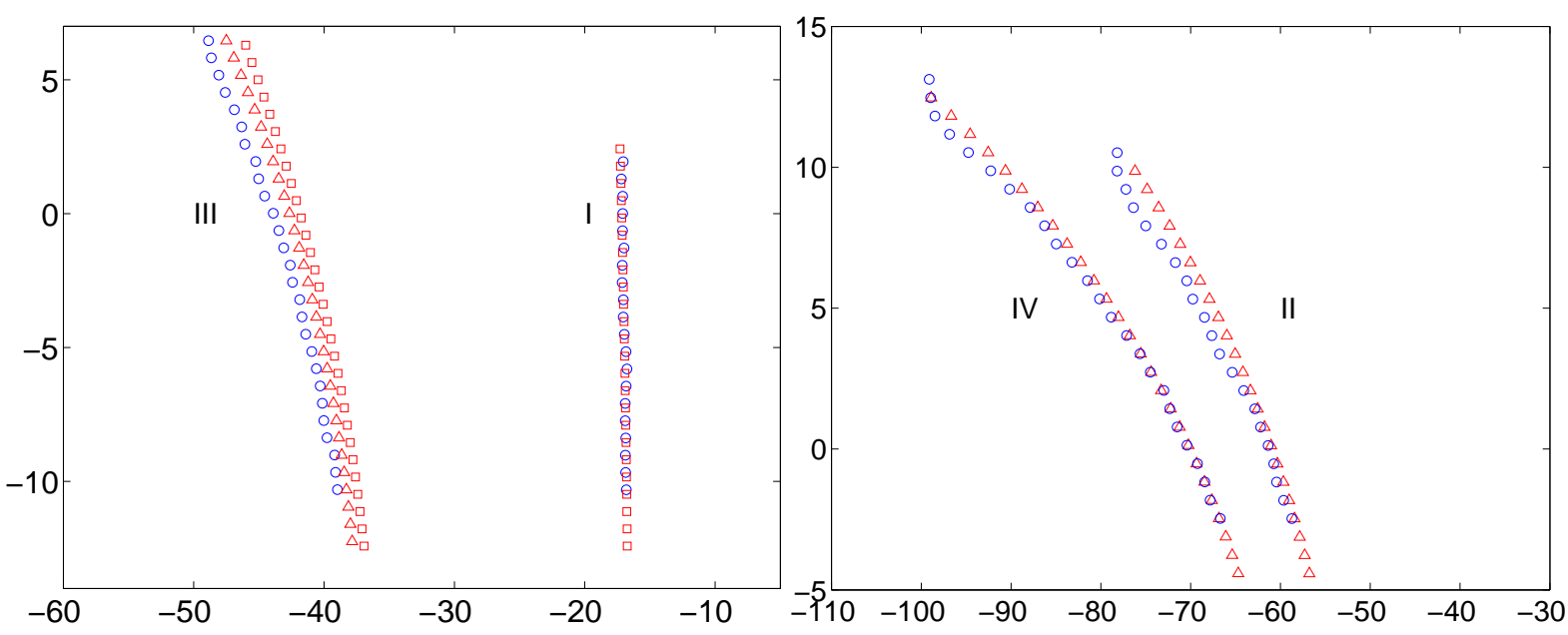

Figure 6: Horisontal velocity profiles under the crest; experiments (circles), Boussinesq (squares) and Tanaka (triangles) solution.

Time gauge measurements are performed also for $h=10 \mathrm{~cm}$. For case IV dimensionless time series are compared to the similar series for $(h=20 \mathrm{~cm}$ ) (figure 8 ). Halving the equilibrium depth causes a relative amplitude reduction equal to 0.04 . This is probably due to viscous damping, that is more pronounced for shallower water. We have no direct measurement of the amplitude variation along the tank and base the assumption on results from the literature. A theoretical model, based on laminar boundary layers, is found in Keulegan (1948). According to this model the attenuation of a soliton becomes

$$
A=A_{0}\left\{1+0.237\left(\frac{A_{0}}{h}\right)^{1 / 4}\left(\frac{\nu c_{0}}{8 h^{3}}\right)^{1 / 2}\left[1+\left(\frac{2 h}{b}\right)\right] t\right\}^{-4},
$$

where $A_{0}=A(t=0), \nu$ is the kinematic viscosity, $c_{0}$ is the linear wave speed and $b$ is the width of the tank. The model does not take turbulence or surface effects into account. Shuto (1976) reported the partly empirical formula

$$
A=\frac{A_{0}}{\left(1+\frac{8}{15} \frac{C_{1} A_{0} x}{h^{2}}\right)}
$$

where $C_{1}$ is a friction coefficient based on measurements and is 0.0227 and 0.0137 for water depth $10 \mathrm{~cm}$ and $20 \mathrm{~cm}$, respectively. Equation (3) implies a stronger damping than (2). In our experiments the wave is not close to a soliton all the way from the paddle. Still, the equation (3) should give a good indication on damping due to viscous and surface effects. Employing (2), with the appropriate parameters for the case IV wave propagating from the paddle to the FOV position, we obtain a reduction of $A / h$ equal to 0.007 and 0.011 for $d=20 \mathrm{~cm}$ and $d=10 \mathrm{~cm}$, respectively. The corresponding results for $(3)$ are 0.055 and 0.086 . In view of the inaccuracies of the surface measurements and the deviations from a perfect soliton shape the latter is in good agreement with the amplitude difference from our wave gauge measurements.

We have observed the following: 

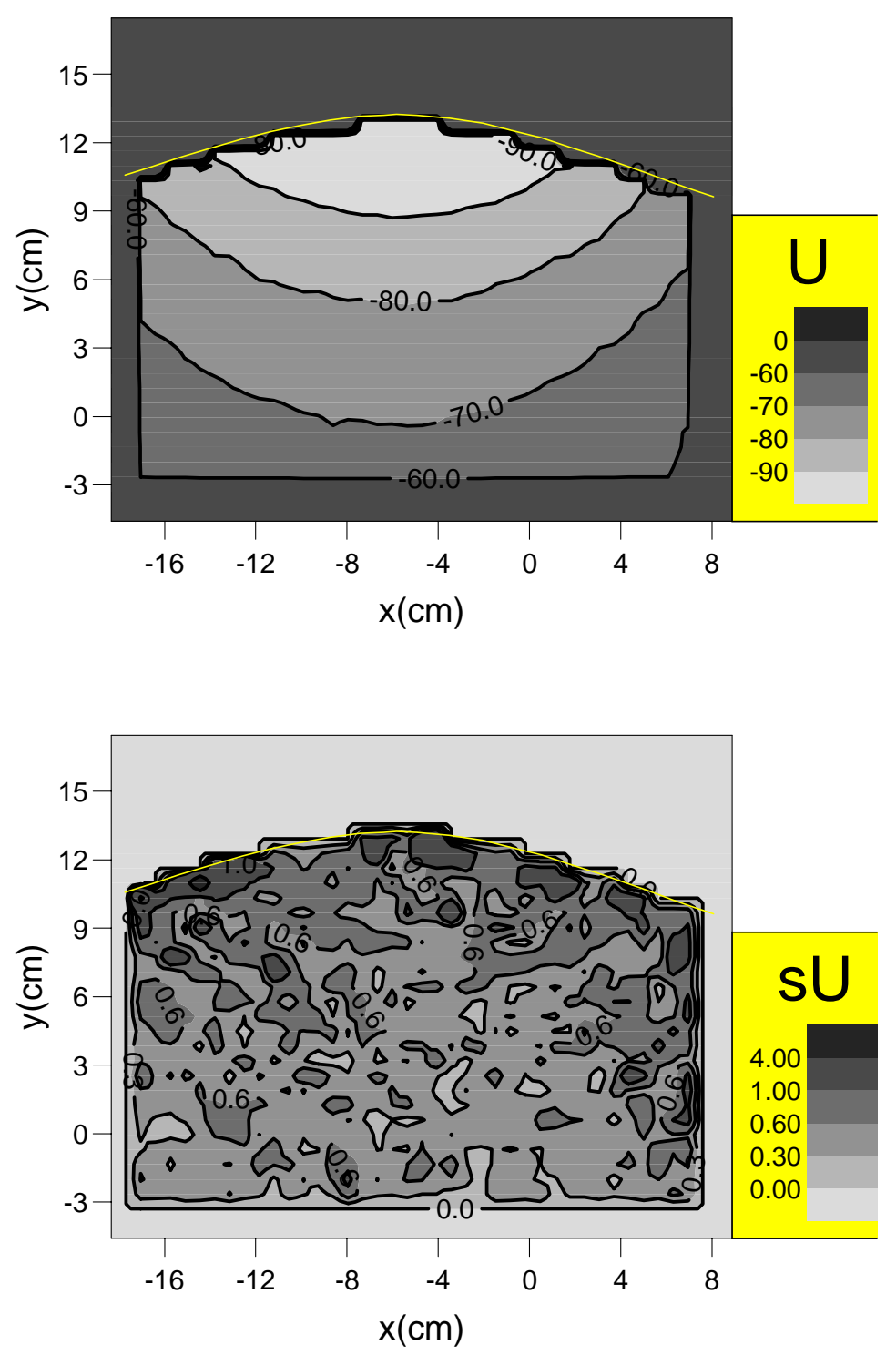

Figure 7: Velocities, in $\mathrm{cm}$ per second, for case $I V$ at $t=2.57 \mathrm{~s}$ as measured by PIV. Upper panel: horizontal velocity, lower panel: standard deviation of horizontal velocity. 


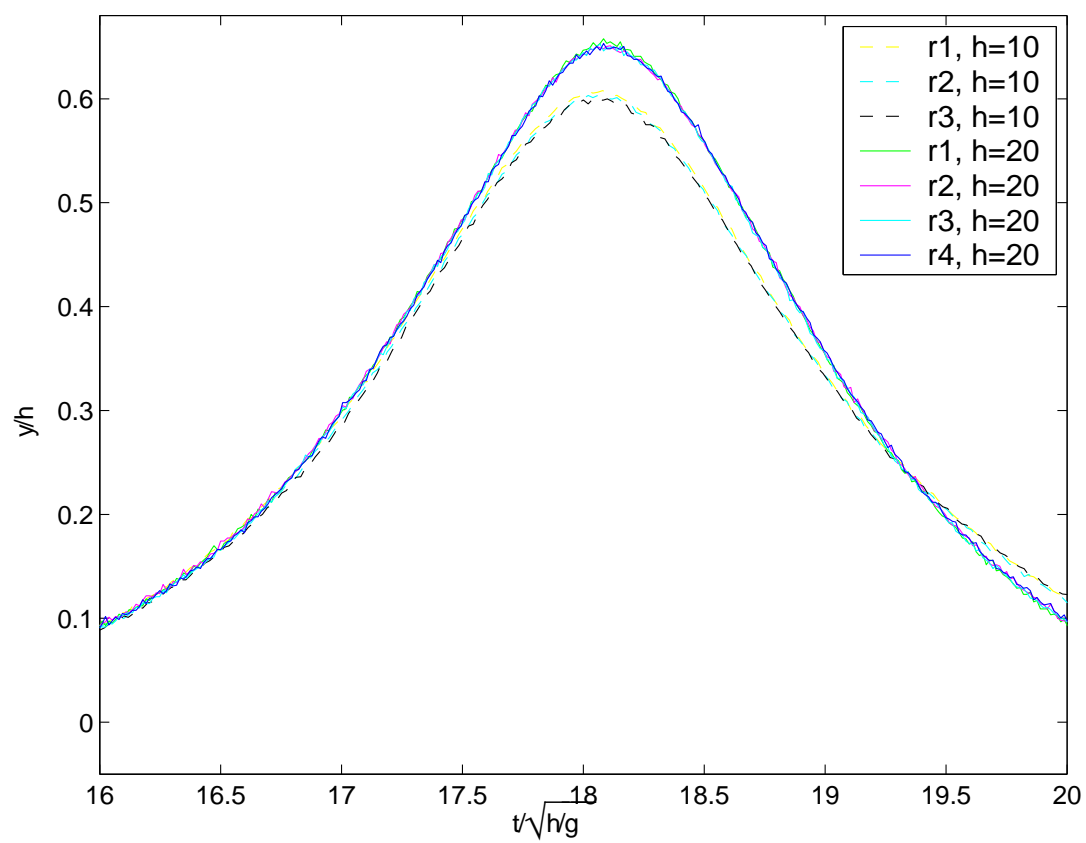

Figure 8: Time gauge measurements for case $I V ; h=10$ and $h=20 \mathrm{~cm}$.

\begin{tabular}{c||l|l|l} 
FOV & $x-\operatorname{span}(\mathrm{cm})$ & $y-\operatorname{span}(\mathrm{cm})$ & cases (and times) \\
\hline \hline (i) & $-15.7<x<1.2$ & $0.9<y<14.5$ & IV $(3.1,3.12)$ \\
(ii) & $-5.5<x<6.1$ & $0.5<y<9.7$ & I $(4.4)$, II $(3.04)$, III $(3.47)$, IV $(3.05)$ \\
(iii) & $-19.5<x<-7.50$ & $3.1<y<12.6$ & II $(3.16)$, III $(3.7)$, IV $(3.14)$ \\
(iv) & $1<x<22.8$ & $-10.6<y<6.9$ & I $(4.55,4.8 .5 .05)$ \\
(v) & $-6.9<x<6.1$ & $-0.15<y<9.9(h=0.1 m)$ & III $(2.51,2.62)$, IV $(2.12,2.18)$
\end{tabular}

Table 3: FOV's in cm and trigger times in seconds.

1. Case II, III and IV are very close to solitons at this position. Reflections from the beach are noticeable only after the first, dominant wave pulse has passed.

2. Table 2 shows that Boussinesq model has lower amplitude for cases IV and II, but both I and III fit well with the experiments.

3. The PIV techniques operate well for the deep water measurements. However, the scatter (figure 7) is sufficiently strong to make straightforward calculation of gradients and accelerations difficult.

\section{Run-up}

We have investigated the run-up of the same four incident waves used in the deep water experiments. PIV measurements were performed with 5 different field of views (FOVs) that were chosen according to the characteristics of the different waves and the wish to identify the physical mechanisms in the first stage of the run-up (table 3). Each experiment was repeated from three to five times. For illustration the run-up of wave 


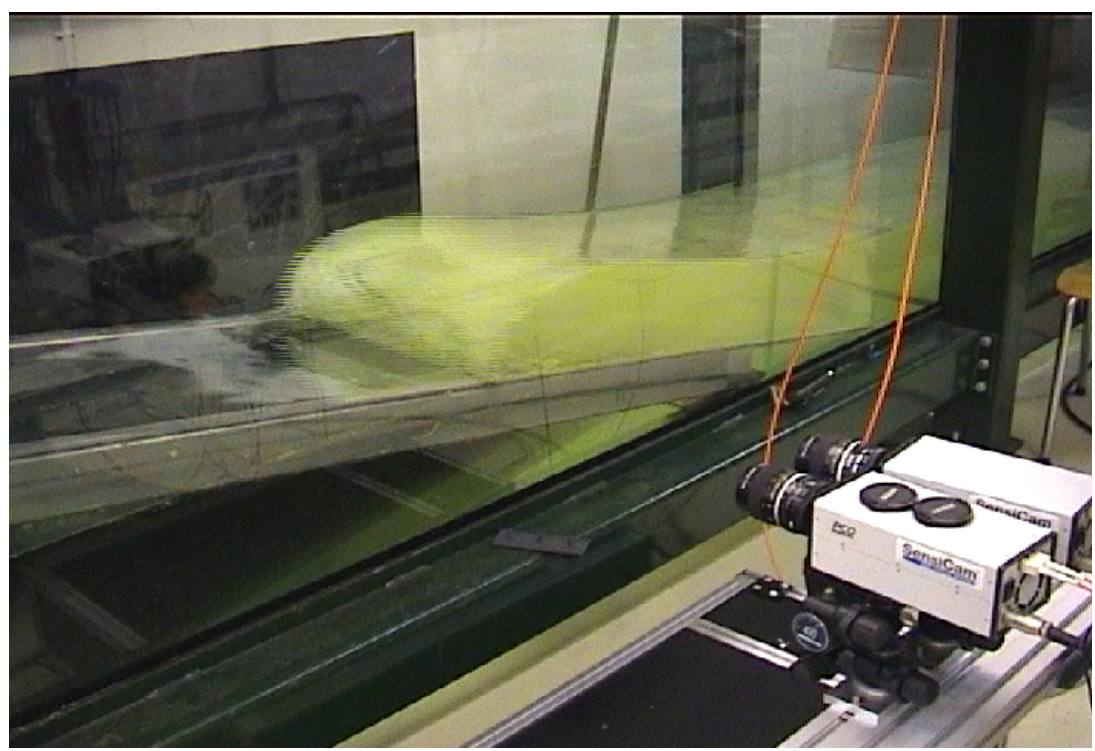

Figure 9: Run-up of wave IV.

IV, together with the camera set-up, is shown in figure 9.

The gentle incident wave (I) should be within the validity range of the Boussinesq model. In figure 10 we have compared surfaces from the experiments with the Boussinesq model. The agreement is very good for $t=4.8 \mathrm{~s}$ while discrepancies are significant for $t=4.4,4.55 \mathrm{~s}$, when the temporal variation of the surface is faster and the Boussinesq solution seems to be delayed. However, it should be noted that the surface at $t=4.4 \mathrm{~s}$ is not accurately extracted, due to light refraction effects in the experiments.

A few selected vertical velocity profiles are depicted in figure 11 and compared to profiles that has been reconstructed from the Boussinesq model (see appendix B). For the horizontal velocities we observe an error, that are systematic in the sense that the Boussinesq solution seems to be $0.05 \mathrm{~s}$ early. If we shift the solution by this time, both magnitude and the weak vertical variation fits very closely to the experiments. This is demonstrated in figure 12, where the distribution of vertically averaged velocities in the FOV is compared. For the much smaller normal velocities the scatter range in the experiments is larger in relative terms, and the Boussinesq solution is within this. In general, the overall agreement is as good as can be expected for case I and very good agreement concerning velocities can be obtained by systematic time shift. Still, simultaneous agreement for velocities and surface cannot be obtained in this manner. Even though the extraction of the free surface is hampered with errors, (in particular for $t=4.4 s$ ) some of the deviations are likely to be due to the inaccuracy of the Boussinesq theory.

For the steeper wave of case III the errors of the Boussinesq equations become significant already in deep water. As shown in figure 13 the deviations for the surfaces are increased, but the agreement is still good. This time we are not able to reproduce the horizontal velocities by introducing a consistent time shift in the Boussinesq solution. According to figure 14 we need a positive time shift for $t=3.47 \mathrm{~s} \mathrm{~s}$, whereas a negative 

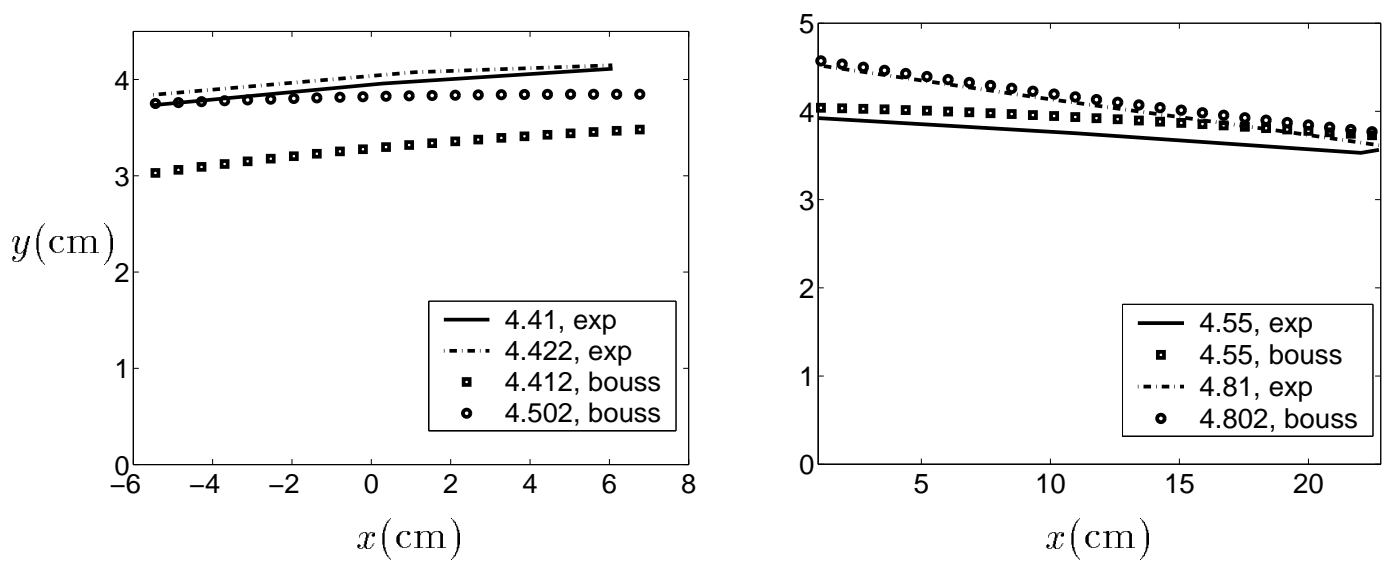

Figure 10: Case I: extracted surfaces from the PIV images compared to the Boussinesq solution.
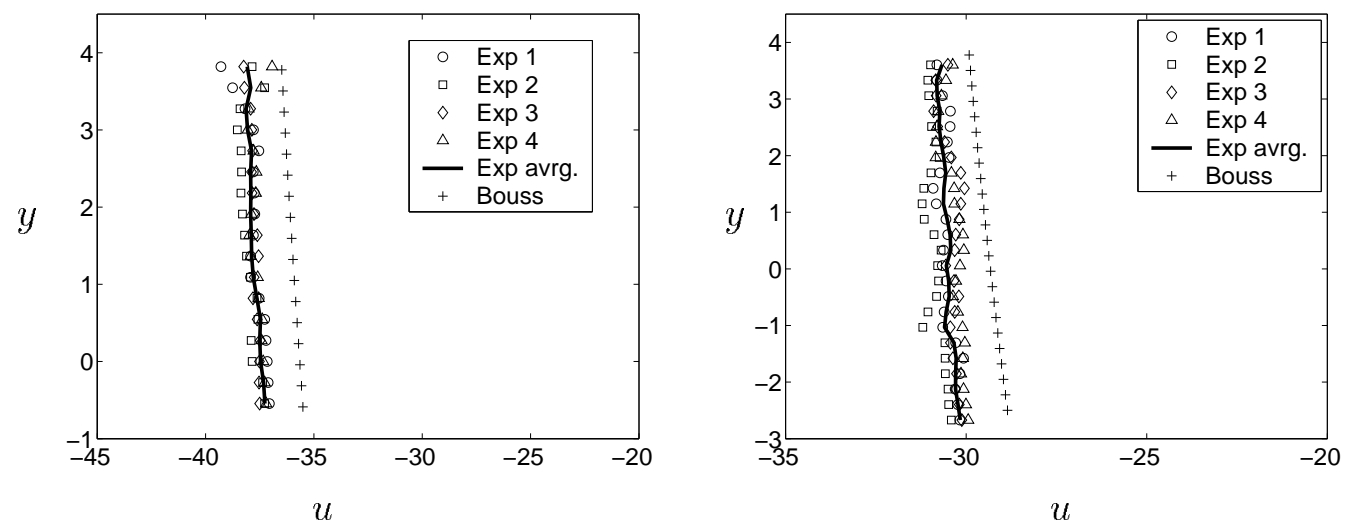

Figure 11: Case $I, t_{t r}=4.55 \mathrm{~s}$ : Velocity profiles of $u$. Left panel: $x=3.5 \mathrm{~cm}$. Right panel: $x=14 \mathrm{~cm}$. 


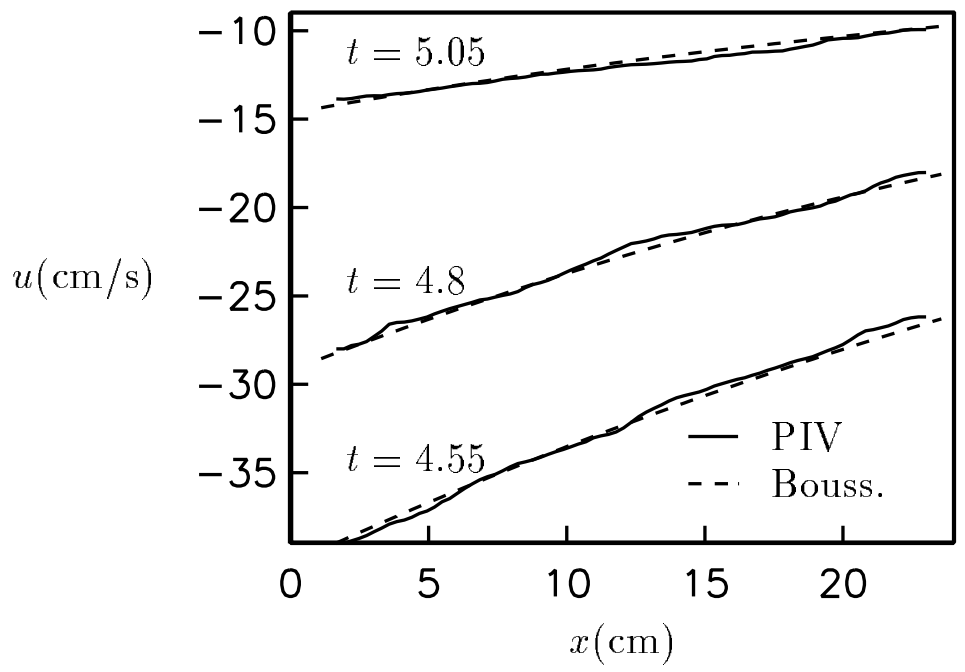

Figure 12: Distribution of vertically averaged $u$ along the FOV. The Boussineq results are consistently shifted $-0.05 \mathrm{~s}$.
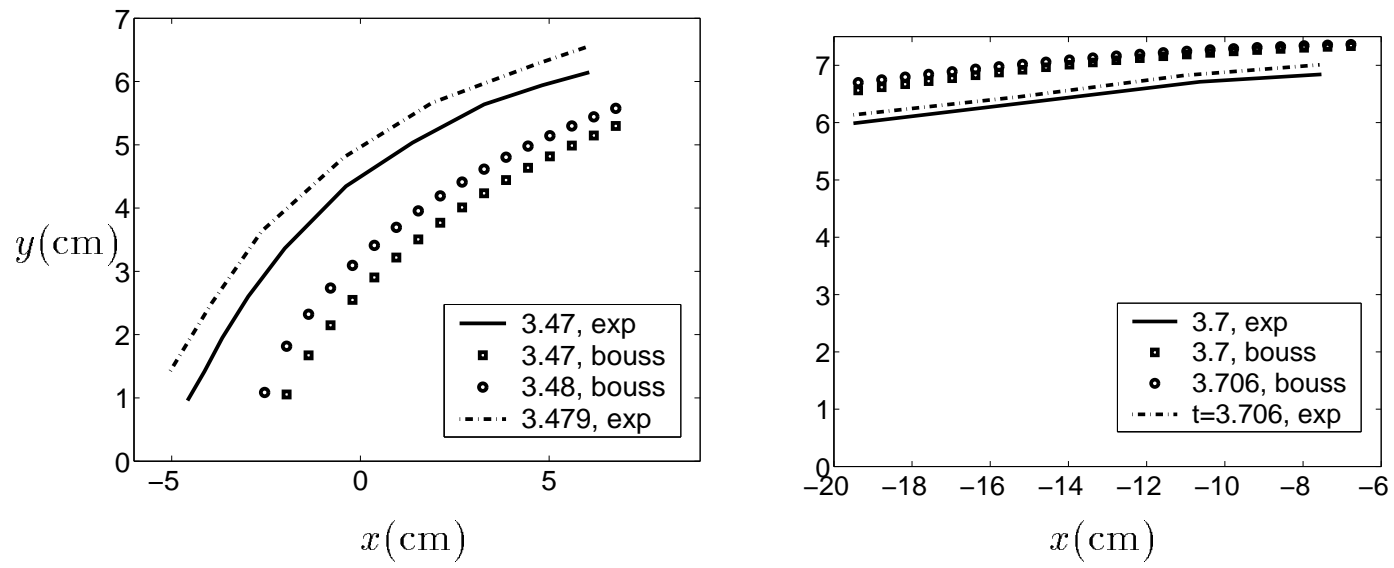

Figure 13: Case III: extracted surfaces from the PIV images compared to the Boussinesq solution. 

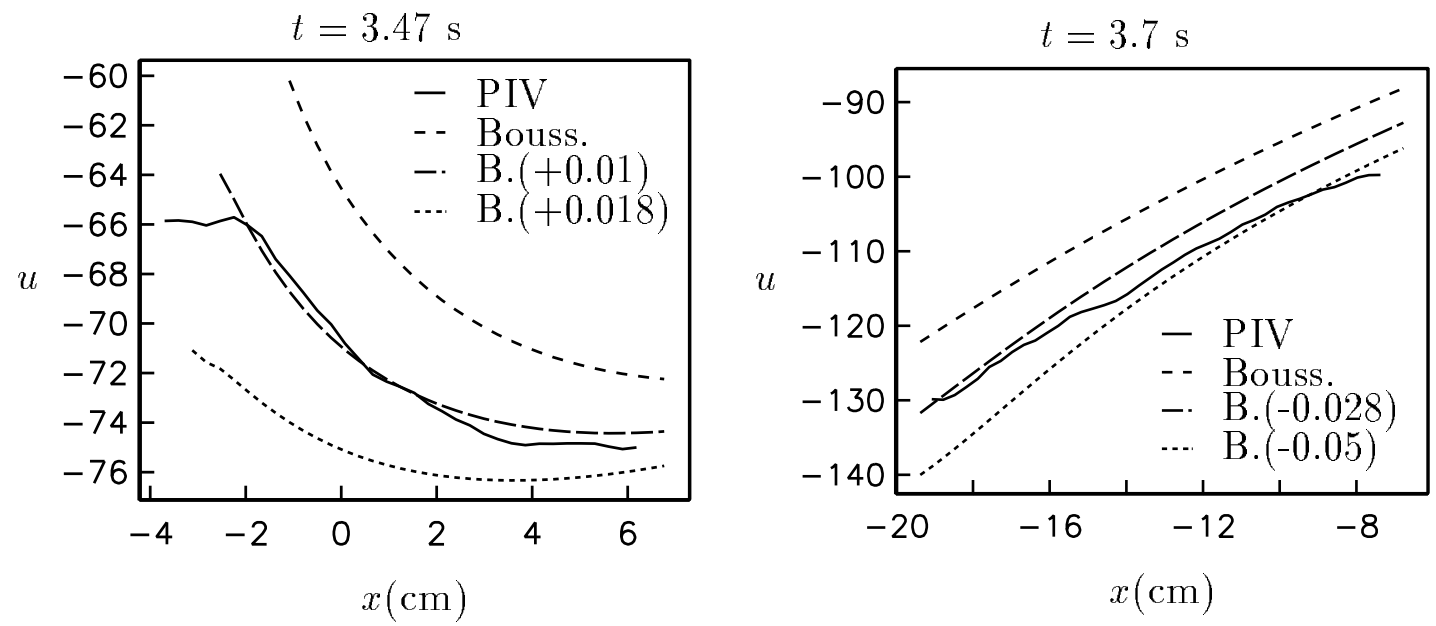

Figure 14: Distribution of vertically averaged $u(\mathrm{~cm} / \mathrm{s})$ along the FOV for case III. The abbreviation B. denotes Boussinesq solution.

shift gives best agreement for $t=3.7 \mathrm{~s}$. Observing the short interval between these two measurements, we realize that the Boussinesq solution, in a sense, evolves much too fast. This can also be inferred from the surfaces in 13 . Moreover, for $t=3.7 \mathrm{~s}$ we do not obtain an accurate horizontal gradient of the vertically averaged $u$. In general the Boussinesq solution is poorer for case III than for case I. Still, the agreement with experiments is fairly good and the qualitative patterns seem correct. It is left open how the errors can be divided between the deep water propagation, that yield erroneous incident wave, and in the run-up phase, respectively.

For the steepest wave, case IV, we will look into the run-up story in quite some detail. During propagation in deep water the incident wave has maximum horizontal velocity near the peak, while the largest horizontal accelerations are found in the wave front, not too far from the position of maximum surface gradient. In shoaling water the front steepens and become shorter, but we have the same qualitative distributions of horizontal velocity and accelerations. However, when the wave reaches the equilibrium shoreline the nature of the motion shifts from being wave-like to become a material fluid body ascending the beach. At this stage the dominant feature of the flow is a translation parallel to the beach slope. We may regard that on top of this there is superimposed a "deforming" velocity field, of smaller magnitude, that determines the evolution of the run up front. We seek to identify and discuss the latter field in the PIV measurements. An image, overlain with the velocity field from PIV, for $t=3.05 \mathrm{~s}$ is shown in figure 15. The wave has just started to climb the beach and we can see an almost vertical front of the wave. Keeping in mind that the translation part of the velocity field is slightly ambiguous, we subtract some value a little behind the front from the measured field. The actual choice is not crucial as long as the residual field provides a clear pattern. A more grave problem is that the errors in the measurements of the large velocities (up to $1.5 \mathrm{~m} / \mathrm{s}$ ) is augmented, in relative terms, when retained in the much smaller residual field (typically $0.1-0.2 \mathrm{~m} / \mathrm{s}$ ). For $t=3.05 \mathrm{~s}$ $\mathrm{s}$, we have displayed the "deforming" wave field in figure 16. Even though the errors are noticeable, the repeatability is still rather good and the standard deviation is generally 

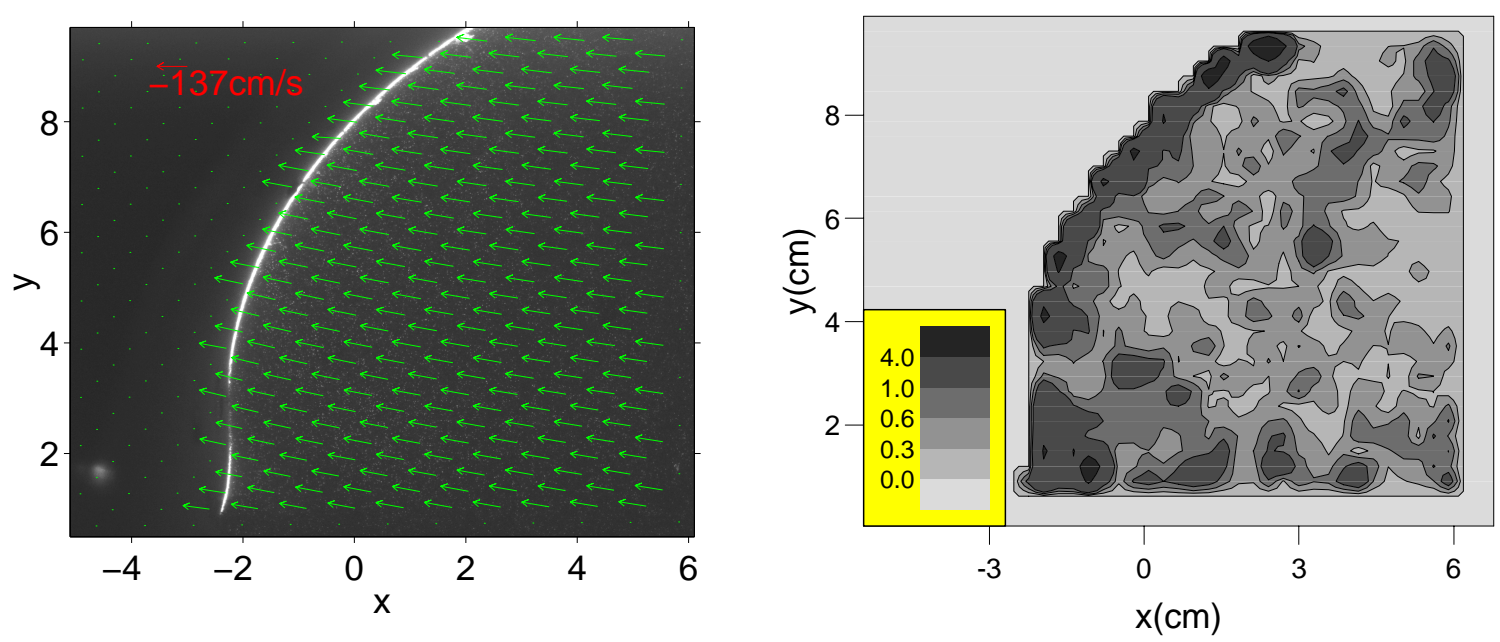

Figure 15: Measured velocities for case $I V$ at $t=3.05 \mathrm{~s}$. Left panel: Vector plot on a PIV-image. Right panel: Standard deviation of $u$ in $\mathrm{cm}$ per second.

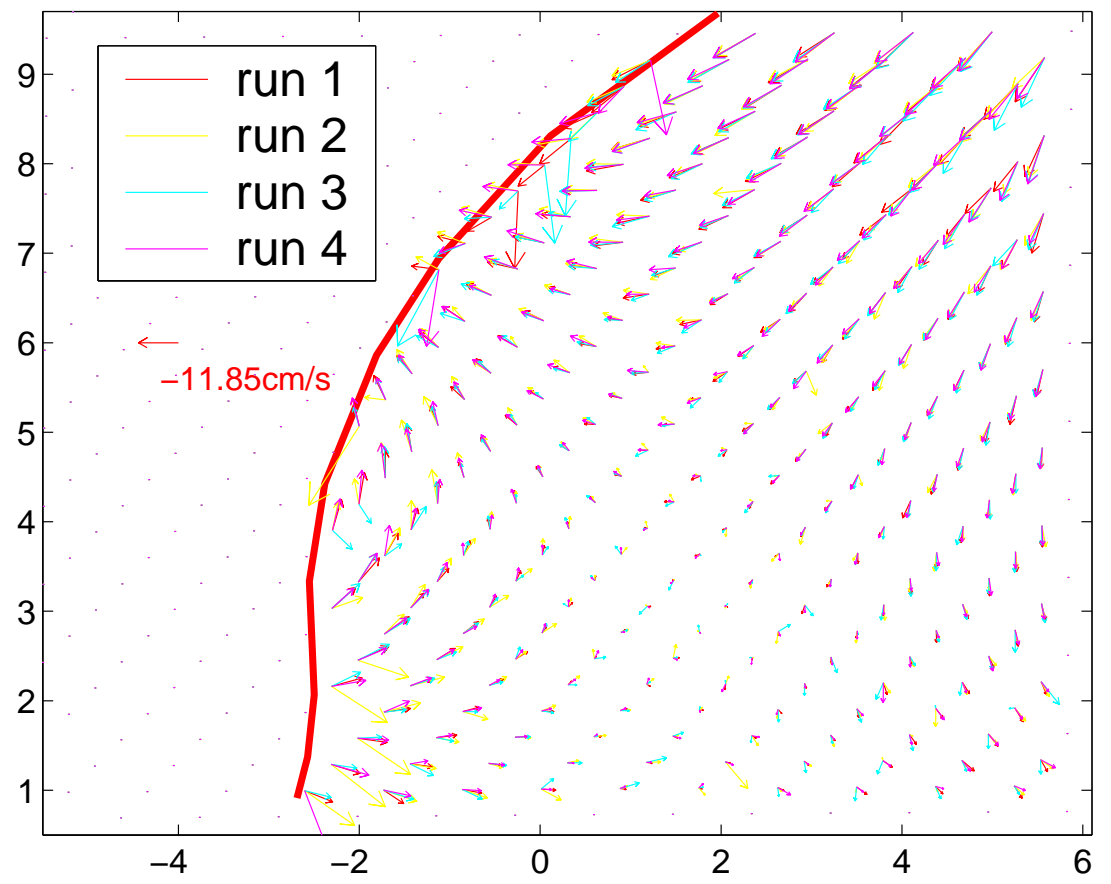

Figure 16: Velocities, subtracted $(-125,23.2) \mathrm{cm} / \mathrm{s}$., for case $I V$ and $t=3.05 \mathrm{~s}$. The different color of arrows correspond to different repetitions of the experiments. 

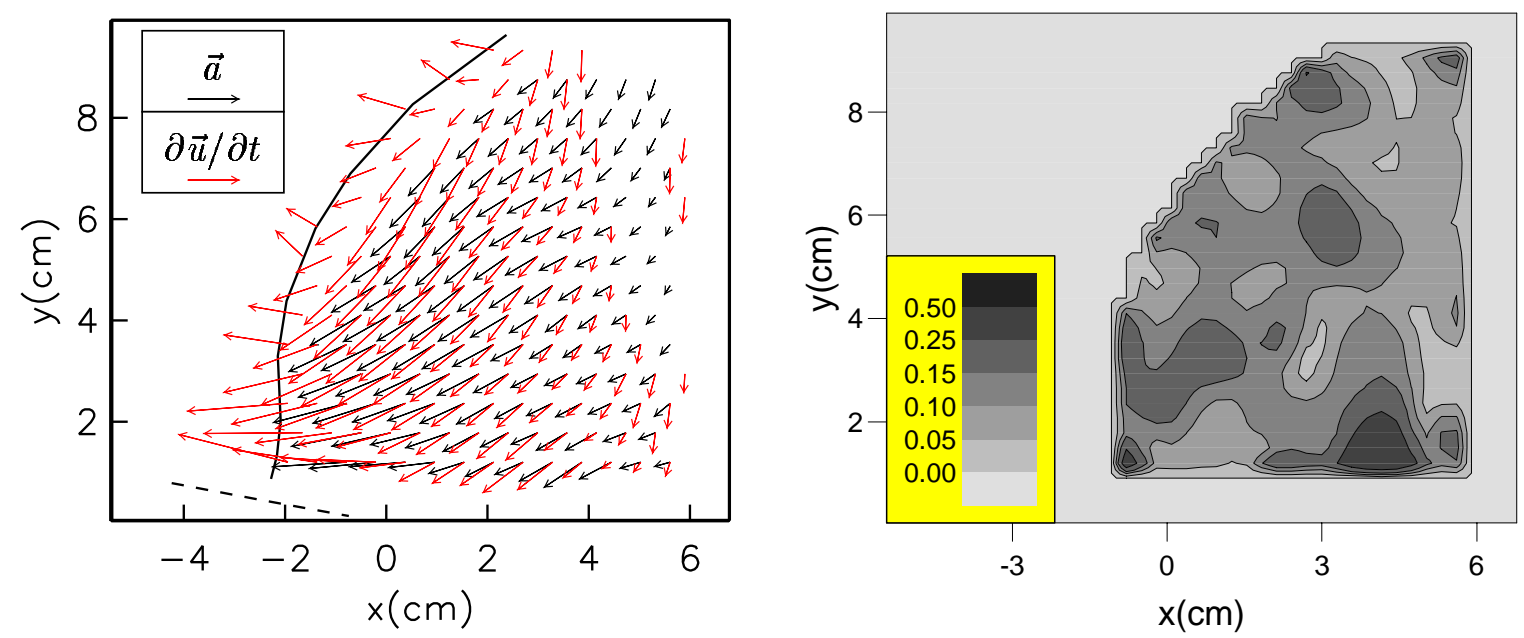

Figure 17: Accelerations for case $I V$ at $t=3.05 \mathrm{~s}$. Left panel: Local and particle accelerations. Reference arrows correspond to $g$. Right panel:Standard deviation of the $x$ component relative to $g$.

less than $1 \mathrm{~cm} / \mathrm{s}$ (figure 15). In figure 16 we clearly see a pattern, inherited from the incident wave, with higher horizontal velocities in the upper region of the wave front as compared to those at the toe. This implies that the front is still steepening. Since the maximum inclination of the surface at $t=3.05 \mathrm{~s}$ is virtually vertical, this means that the wave front will be overturning shortly. In fact, this is observed in other images. Still no clear signs of breaking are visible in the video recordings or subsequent PIV images. This is due to the acceleration distribution. Accelerations are computed from the PIV velocities by a tracing technique, as explained in appendix A, and displayed in figure 17. Certainly, the accelerations are (at least) as sensitive to experimental scatter as the velocity "residual" discussed above. However, even though there are errors (see figure 17, right panel), and no accelerations are measured in the very vicinity of the fluid surface, we clearly observe a distinct pattern with large, mainly horizontal, velocities behind the toe (larger than $g$ ), while the accelerations close to the top of the run-up front are much smaller, and with a strong downward component. This picture is very different from the one reported by New et al. (1985) for breaking waves in deep water. In that case the accelerations at the base of the wave front is moderate and directed upward and forward relative to direction of wave advance. As the particle travels up the wave front the horizontal component increases, whereas the vertical component change sign. Obviously, the run-up acceleration pattern in figure 17 opposes the development of breaking and indicates a tendency toward thinning of the fluid front. Consequently, at the slightly later time $t=3.12 \mathrm{~s}$ (figure 18) the overall picture of the flow has changed; we observe the embryo of the on-shore jet (see introduction) with a clear stretching of the fluid tongue according to the "deformation" velocity field. The evolution of this early stage in the run-up is rapid, due to huge accelerations possessing strong gradients. The occurrence of breaking during run-up is then determined by the competition between two features, namely the velocity distribution conveyed from the incident wave, that points to breaking, and the opposing acceleration field. Breaking, then occurs when the vertical shear in the horizontal velocities is so strong that the 


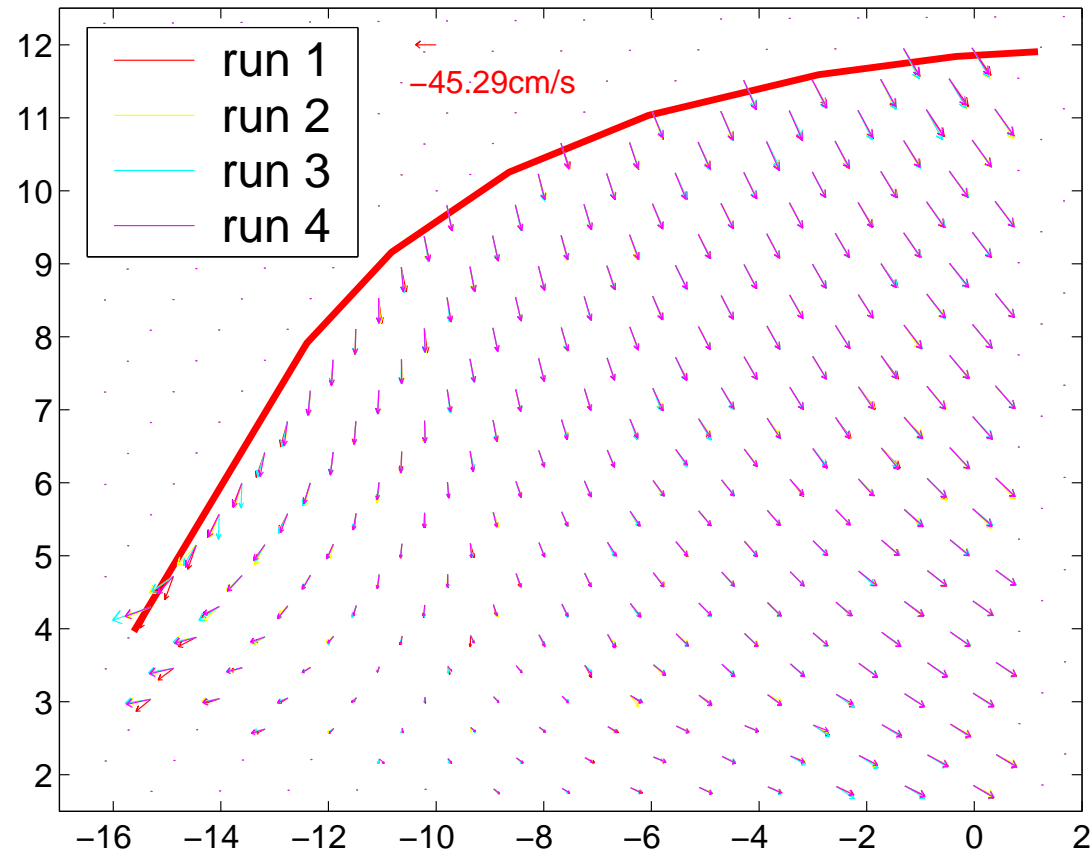

Figure 18: Velocities, subtracted $(-170,31.5) \mathrm{cm} / \mathrm{s}$, for case $I V$ and $t=3.12 \mathrm{~s}$.

overturning becomes too far advanced before the accelerations are able to reverse the shear.

A few additional comments should be attached to the run-up of a nearly breaking wave. First we have the analogy to the classical dam-break problem (Stoker (1957)). This analogy is also mentioned by Peregrine \& Williams (2001). To make this link clearer we introduce a coordinate system with axes parallel and normal to the beach plane. Moreover, at a time, when the fluid front is nearly vertical, we choose the initial velocity of the system equal to the particle velocity at the instantaneous shoreline, while the acceleration of the system is specified as the component of gravity parallel to the slope. We then end up with a formulation of an initial value problem with a steep fluid front, nearly at rest, and an external force field (gravity + fictitious) normal to the bottom. The major difference from the standard dam-break problem is the presence of velocities behind the front. For an ideal fluid the dam-break problem yields large accelerations at the bottom of the fluid front. If the contact angle between fluid and bottom is $90^{\circ}$, or larger, we obtain in fact a singularity. The qualitative agreement with the accelerations in figure 17 is striking (see figure 23). Naturally, the presence of the thin viscous boundary layer in the experiments prevents too extreme accelerations. We are not able, anyhow, to measure very close to the fluid boundary. The dam-break analogy is somewhat elaborated in appendix C.

From the acceleration measurements we may also infer that a long wave approximation, even a higher order one, is not likely to be valid at the crucial stage in run-up, when the on-shore jet is initiated. This is seen from the large vertical accelerations in figure 17 as well as the profiles in figure 19 that are both irreconcilable with long wave theory. Indeed we find large discrepancies between the Boussinesq model and the experiments for case IV. However, this is also due to errors in the generation and propagation in deep water that yield a far too long incident wave with too small amplitude in the Boussinesq computation. Hence, in spite of the observed deviations it 
is still possible that a combination of "full" theory in the deeper region and a long wave model close to the shore may be appropriate. Combined models of this kind, but with a linear offshore region, could be attractive for modeling of tsunamis and ocean swells. Unfortunately, our experiments indicate that such models have poor prospects for the computation of waves that become very steep at the shore. This is supported by the analysis of appendix B. The computational aspects will be pursued further in a forthcoming paper.

The slightly gentler case II does not differ very much from IV. In the run-up zone the wave is less steep but the quantitative patterns for the velocities and accelerations are the same. The acceleration measurements show also the formation of a jet in similar matter as case IV (results not shown).

In principle the Boussinesq solution breaks during run-up for case II and IV. However, the breaking occur only with grid resolutions much finer than what is needed for the convergence of all other characteristics (see appendix B). For case IV breaking in the solution occur shortly after $t=3.05 \mathrm{~s}$. Even though there now must be large errors in both incident wave and run-up we still observe a fair agreement with experiments at this time, with an obvious delay of the Boussinesq data. Horizontal velocity profiles are shown in figure 19 .
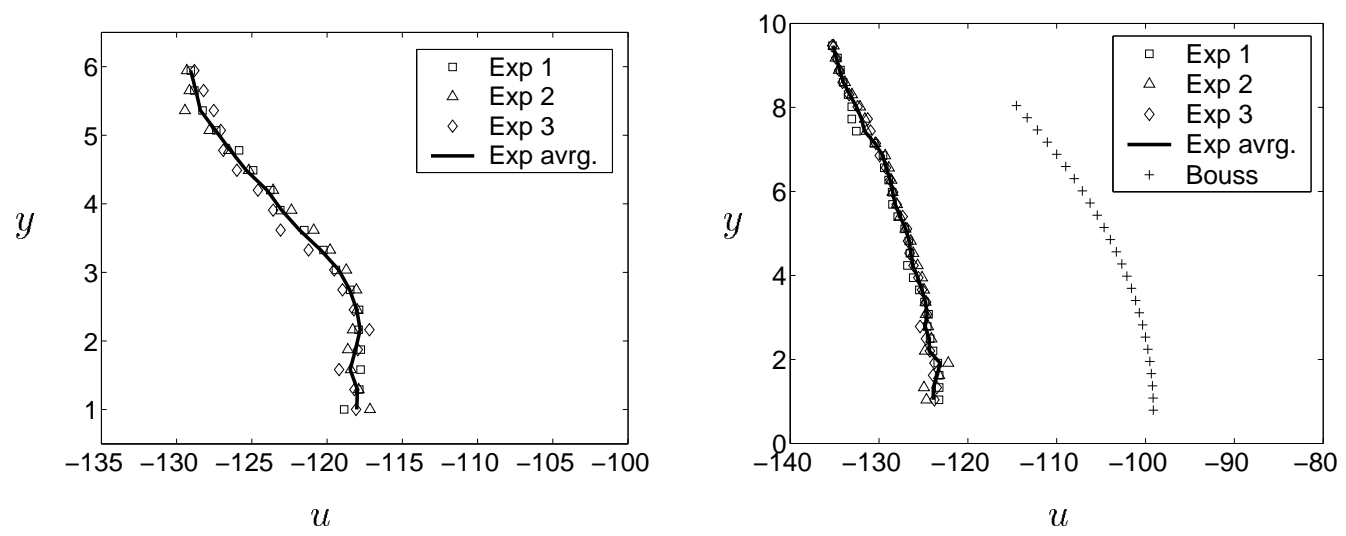

Figure 19: Case IV, $t_{t r}=3.05 \mathrm{~s}:$ Velocity profiles of $u$. Left panel: $x=-1.5 \mathrm{~cm}$. Right panel: $x=4 \mathrm{~cm}$.

We have also measured the run-up heights, by direct eye observation, for all cases with depth $h=20 \mathrm{~cm}$ and case III and IV for $h=10 \mathrm{~cm}$. For the steeper cases, II and IV, some difficulty was associated with the three dimensional effects, that manifested themselves as a curved front with arbitrary narrow fingers sticking out (see figure 20), that were neglected when run-up heights were estimated. The subjectivity of the method employed, combined with the slightly ambiguous definition of fluid front for case II and IV, points to significant errors. Still, the results were repeatable, within a $\mathrm{cm}$. One result that may seem surprising, is the little variation of the ratio $R / A$ (run-up height to amplitude of incident waves) with steepness of incident the wave, see table 4. This is in contrast to the Boussinesq results, that are generally higher and with $R / A$ increasing with $A$. The discrepancy may partly be due to viscosity and 


\begin{tabular}{r||c|c|c||c|c|c} 
case & $\operatorname{EXP}(R, 20 \mathrm{~cm})$ & $\operatorname{EXP}(R, 10 \mathrm{~cm})$ & $\operatorname{Bouss}(R)$ & $\frac{R}{A}(\mathrm{~B})$. & $\frac{R}{A}(20)$ & $\frac{R}{A}(10)$ \\
\hline \hline IV & $41.2 / 20=2.1$ & $19.3 / 10=1.9$ & $50.1 / 20=2.5$ & $4.5^{*}$ & 3.1 & 3.1 \\
III & $23.7 / 20=1.2$ & $10.6 / 10=1.0$ & $27.2 / 20=1.4$ & $4.1^{*}$ & 3.4 & 3.1 \\
II & $35.4 / 20=1.77$ & - & $39.5 / 20=2.0$ & 4.3 & 3.3 & - \\
I & $8.8 / 20=0.4$ & - & $9.3 / 20=0.5$ & 3.7 & 3.5 & -
\end{tabular}

Table 4: Run-up height; EXP - experiments and Bouss (B.) - Boussinesq simulations. The values marked by ${ }^{*}$ are uncertain in the sense that grid refinement eventually leads to breaking. See discussion in appendix.

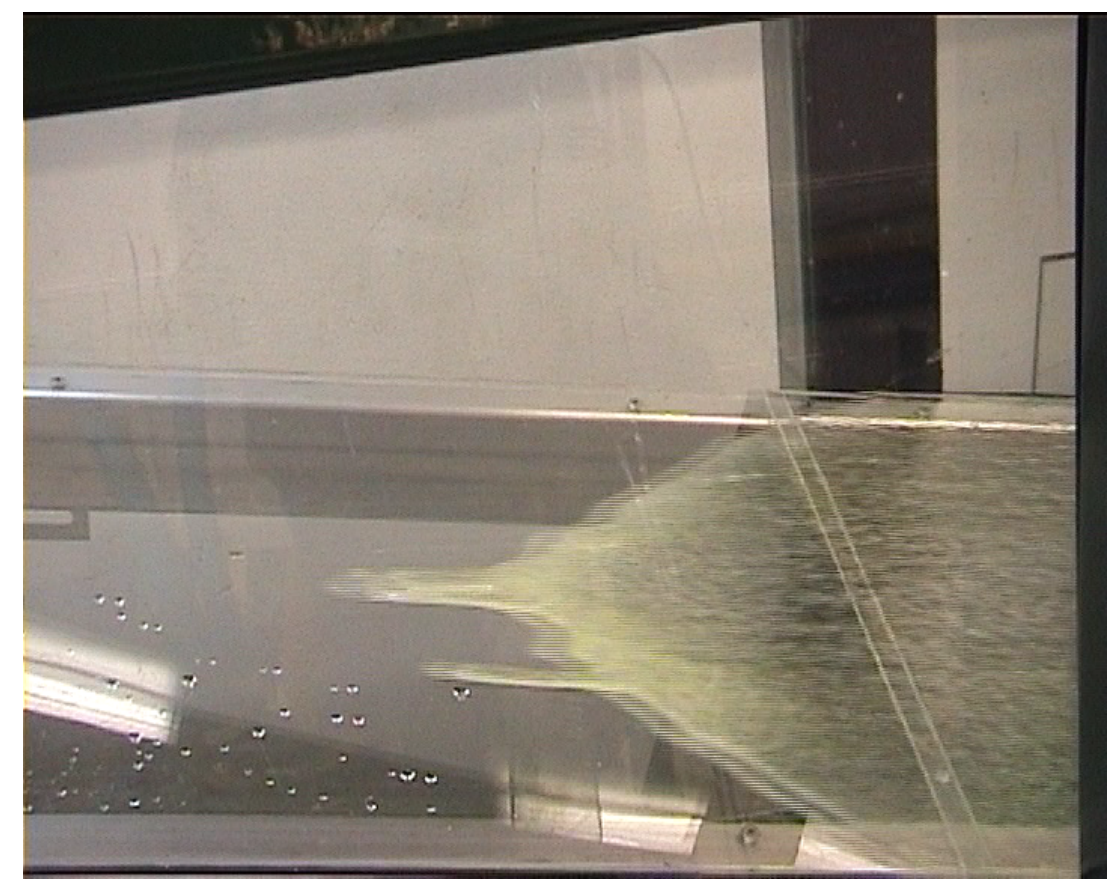

Figure 20: Image from the run-up of Case $I V$.

cappilary effects, that are important on laboratory scale, particular in the thin upper part of the run-up tongue. In accordance with this we find a marked scale dependence for case III and good agreement between the Boussinesq model and experiments for case I, where the the incident wave is long and the run-up tongue comparatively thick. Surprisingly, we observe no scaling effect on the run-up height for case IV. This may be due to a cancellation of scaling effects in incident wave, run-up tongue and the early crucial stages of the run-up. However, at present we have insufficient information to make any firmer analysis. For the steeper cases, II and particularly IV, the runup is not strictly two-dimensional, but displays a kind of fingering (figure 20). The mechanism behind this phenomenon has not been investigated closely in the present study, but the fact that it is observed for the steeper incident wave points to the possible evolution of three dimensional features in the early run-up stages. We have also tried to discover three-dimensional patterns by extracting divergence from the two-dimensional experimental velocity fields. These attempts failed because of noise in the results from the calculations of gradients. 

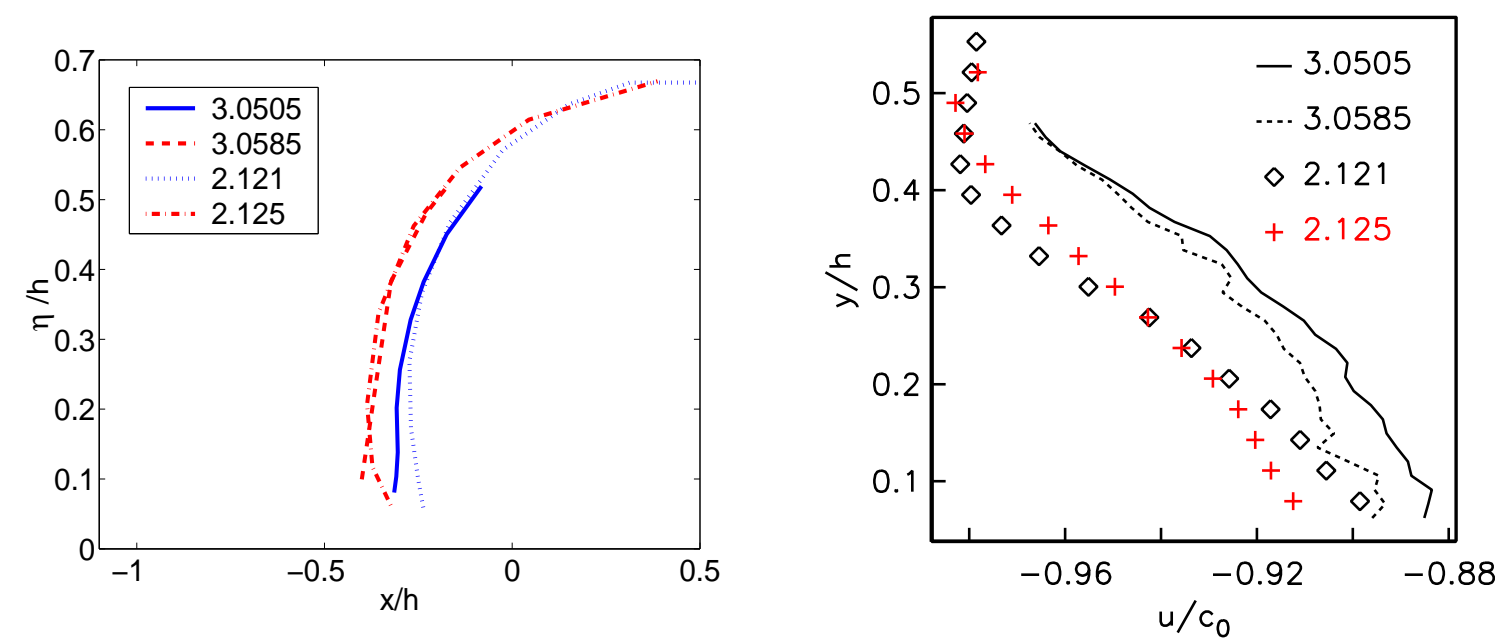

Figure 21: Case $I V, t_{t r} \approx 3.05 \mathrm{~s}(h=20 \mathrm{~cm})$ and $t_{t r} \approx 2.12 \mathrm{~s}(\mathrm{~h}=10 \mathrm{~cm})$. Left: Surface profiles. Right: Velocities. The upper region of nearly constant $u$, observed for $t_{t r}=2.121 \mathrm{~s}$ is due to experimental errors near the surface. The remainder of this profile, as well as almost the whole profile for $t_{t r}=2.125 \mathrm{~s}$, is measured accurately with good repeatability.

Scaling effects on other properties were also checked for case IV. Surface profiles and horizontal velocities were extracted from the experiments with depth $h=20 \mathrm{~cm}$ and compared with $h=10 \mathrm{~cm}$. The left panel in figure 21 shows some minor deviations in the surface profile at the "toe" of the wave. An explanation to this may be that the boundary layer affects the propagation at the beach when the depth is $h=10 \mathrm{~cm}$. The difference in the horizontal position is not large, $\Delta x / h \approx 0.07$, and can be caused, partly, by the accuracy of the trigging. The right panel shows a difference in the velocities of about $4 \%$. However, the profile has somewhat similar features close to the beach as for the surface profiles.

\section{Concluding Remarks}

Apart from the very vicinity of the free surface we have made good velocity measurements with a high degree of repeatability, even during the rapidly evolving run-up. Accelerations, on the other hand, are obtained only under favorable conditions, and then with limited accuracy. Still, important information is present in the few acceleration fields that have been found.

In deep water we found good agreement between our experiments and theory. The main focus of the present study is the kinematics of the waves running up the beach. Our longest incident wave (case I), was reproduced very closely by the Boussinesq model. For the moderately short and soliton-like case III, with amplitude/depth $=0.33$ in deep water, the Boussinesq model still performed well, though the deviations were significant.

For the highest incident wave (case IV, amplitude/depth $=0.66$ in deep water ) the wave front becomes very steep. In a short period of time it is in fact slightly overturning. Still, the wave does not proceed to breaking. Measurements show an 
acceleration pattern that opposes the breaking process and points to the formation of an on-shore jet close to the bottom. The analogy to the dam-break problem is interesting. It is plausible that a similar mechanism is present in the run-up of a fully developed bore. Moreover, it is not likely that any long wave model can reproduce this crucial stage in run-up.

Some effects of scaling was observed, but it was not substantially larger in the early stages of run-up than in deep water.

Pronounced three dimensional effects were found for the higher incident waves. However, the explanation and systematic measurement of these is beyond the scope of this paper.

Acknowledgments. This work was conducted under the Strategic University Programme 'General Analysis of Realistic Ocean Waves' funded by the Research Council of Norway. The discussions with J. Kristian Sveen, Didier Clamond and John Grue, and the technical assistance by Arve Kvalheim and Svein Vesterby are gratefully acknowledged. 


\section{A Smoothing, accelerations}

Even though good accuracy and a high degree of repeatability are obtained in most of the PIV measurement, there are still small errors, with rapid spatial and temporal fluctuations, that render extraction of gradients and accelerations difficult. Hence, we have been able to compute accelerations from PIV data only for the cases where we have the largest accelerations compared to noise level of the velocity fields.

The first step toward determination of gradients is smoothing. Some smoothing, within the interrogation domain, is inherent in the PIV algorithm (see for example Raffel et al. (1998)). On the resulting PIV velocities we apply a very simple three point smoothing scheme that reads

$$
u_{j}^{*}=\frac{1}{4} u_{j-1}+\frac{1}{2} u_{j}+\frac{1}{4} u_{j+1}
$$

where $j-1, j$, and $j+1$ are the numbers of three adjacent points along a line in the velocity matrix. The smoothing is applied subsequently for both grid directions and the procedure is repeated, generally 2-4 times. At the outskirts of the domain with computed velocities, where $u_{j-1}$, say, is not available, the above formula is replaced by the asymmetric version:

$$
u_{j}^{*}=\frac{3}{4} u_{j}+\frac{1}{2} u_{j+1}-\frac{1}{4} u_{j+2} .
$$

The accelerations have been computed in two ways from the subsequent, smoothened, velocity fields $\vec{v}(x, y, t)$ and $\vec{v}(x, y, t+\Delta t)$. First, finite differences can be applied to calculate all derivatives needed to evaluate the material derivative of the acceleration $\vec{a}=\partial \vec{v} / \partial t+\vec{v} \cdot \nabla \vec{v}$. The alternative approach, that is preferred herein, is to trace imaginary fluid particles over the time span $\Delta t$. Denoting the position and velocity of a particle at $t$ by $\vec{r}_{1}$ and $\vec{v}_{1}$, respectively, we employ a mid-point integration of position with time to obtain the implicit expression

$$
\vec{v}\left(x_{2}, y_{2}, t+\Delta t\right)=\vec{v}_{2}, \quad \vec{r}_{2}=\vec{r}_{1}+\frac{\Delta t}{2}\left(\vec{v}_{1}+\vec{v}_{2}\right)
$$

where $\vec{r}_{2} \equiv\left(x_{2}, y_{2}\right)$ and $\vec{v}_{2}$ are the position and velocity at time $t+\Delta t$. Velocities at locations not coinciding with grid points are generally found by bilinear interpolation, or by linear interpolation within triangles close to the fluid margin. Equation (6) is solved by a simple iterative technique, where new $\vec{r}_{2}$ and new $\vec{v}_{2}$ are computed alternatively. Generally, two iterations suffice. Finally the acceleration is calculated by the difference

$$
\vec{a}=\frac{1}{\Delta t}\left(\vec{v}_{2}-\vec{v}_{1}\right)
$$

Naturally, this approximates most closely the value at position $\vec{r}=\frac{1}{2}\left(\vec{r}_{1}+\vec{r}_{2}\right)$ and time $t+\frac{1}{2} \Delta t$. However, the error by assigning it to the coordinates $\vec{r}_{1}$ and $t$ is small compared to the amplified errors inherited from the velocity fields.

\section{B Long wave equations}

The experiments have been compared to, and partly verified by, solutions of a set of Lagrangian Boussinesq type equations described in Pedersen \& Gjevik (1983). Even 
though the numerical technique differs somewhat from the one given in the reference, we omit the details and refer some key results for completeness only.

Following the usual convention of using a typical depth $h_{0}$ and a wavelength $L$ as vertical and horizontal length scales, respectively, and $\left(g h_{0}\right)^{\frac{1}{2}}$ as velocity scale, we may write the continuum and momentum equations according to

$$
\begin{aligned}
& \frac{\mathrm{D} H}{\mathrm{~d} t}=-H \frac{\partial \bar{u}}{\partial x}, \\
& \frac{\mathrm{D} \bar{u}}{\mathrm{~d} t}=\frac{\mathrm{d} h}{\mathrm{~d} x}-\frac{\partial H}{\partial x}-\frac{\beta}{3}\left[H \frac{\partial}{\partial x}\left(\frac{\mathrm{D}^{2} H}{\mathrm{~d} t^{2}}\right)+2 \frac{\partial H}{\partial x} \frac{\mathrm{D}^{2} H}{\mathrm{~d} t^{2}}\right],
\end{aligned}
$$

where $H \equiv h+\eta$ is the total water depth, $\bar{u}$ is the depth averaged horizontal velocity, $\beta \equiv h_{0}^{2} / L^{2}$ is a measure of dispersion and $\frac{\mathrm{D}}{\mathrm{d} t} \equiv \frac{\partial}{\partial t}+\bar{u} \frac{\partial}{\partial x}$ is the material derivative. Often, Lagrangian descriptions become rather messy. However, this is not true in the present case due to the nearly one dimensional nature of the flow. We obtain the Lagrangian form of the equations simply by recognizing the material derivative as the partial temporal derivative and replace $\frac{\partial}{\partial x}$ by $\frac{H}{H_{0}} \frac{\partial}{\partial a}$, where $a$ and $H_{0}$ are the reference position and height of the material water columns, respectively. It is noteworthy that transforming the $x$ derivative applied to $x$ itself yields the continuum equation in terms of the displacements.

Use of the Lagrangian formulation has the advantage that the computational domain is fixed. At the shoreline point, that is associated with a fixed value of $a$, we employ the condition $H=0$. Correspondingly, the fluid column in contact with the wave paddle is also recognized by a fixed $a$. At this location the measured velocity of the laboratory paddle is imposed.

When $(8,9)$, or rather their Lagrangian counterparts, have been solved numerically, the requirements of zero divergence and rotation may be used to restore the vertical variations in the velocity fields according to

$$
\begin{aligned}
& v(x, y, t)=\beta^{\frac{1}{2}}\left(\frac{\mathrm{D} \eta}{\mathrm{d} t}+\frac{\partial \bar{u}}{\partial x}(\eta-y)+O(\beta)\right), \\
& u(x, y, t)=\bar{u}+\beta\left(A_{1}(y-\bar{y})+A_{2}\left(y^{2}-\overline{y^{2}}\right)\right)+O\left(\beta^{2}\right), \\
& A_{1}=\frac{\partial}{\partial x}\left(\frac{\mathrm{D} \eta}{\mathrm{d} t}+\eta \frac{\partial \bar{u}}{\partial x}\right), \quad A_{2}=\frac{\partial^{2} \bar{u}}{\partial x^{2}}
\end{aligned}
$$

To evaluate these expressions at the appropriate spatial and temporal position we apply an extra set of interpolation and differencing applied to the numerical solution. However, grid refinement tests are performed to assure that the total discretization errors are negligible, apart from the very vicinity of the instantaneous shoreline.

For each case the Boussinesq model is run with a series of resolutions, ranging from 160 to 2400 points covering the whole wave tank. Close convergence is apparently obtained already for the coarsest of these resolutions. However, even though global convergence is obtained the maximum steepness under run-up still in increases with resolution for case II and, in particular, IV. For a given resolution we then reach $\partial x / \partial a=0$, that may be used as breaking criterion in the Lagrangian description. It is tempting to assume that quantities like run-up heights obtained from the somewhat coarser grids still may be of significance. However, according to appendix $\mathrm{C}$ this is doubtful since the Boussinesq equations do not reproduce the behavior of steep 


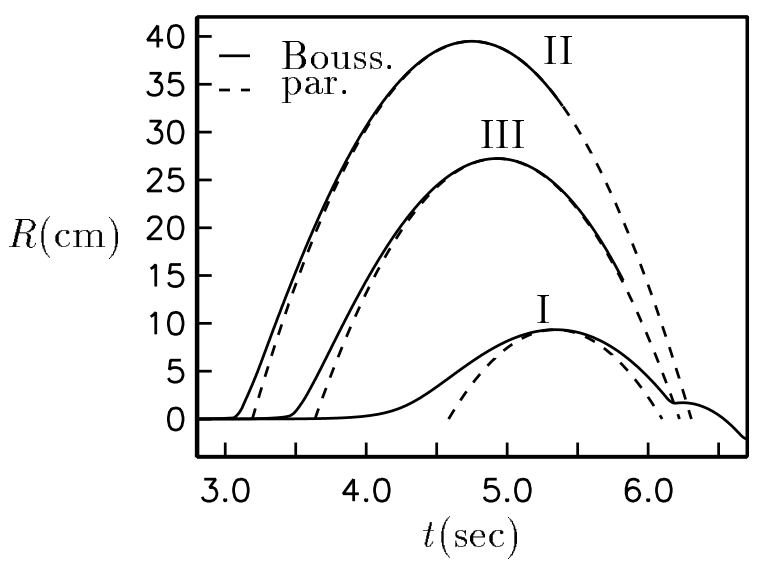

Figure 22: Run-up heights (cm) from Boussinesq model as function of time (seconds) for case I through III. The numerical results are compared to the "parabola" solution as explained in the text.

wave fronts properly. Still, some run-up results for the steeper cases are presented for completeness.

Assuming that gravity dominates in the run-up tongue we may anticipate that the parabola approximation

$$
R \approx R_{\max }-\frac{1}{2} g \sin \theta \cos \theta\left(t-t_{m}\right)^{2}
$$

is valid around the time of maximum run-up, $t_{m}$. According to figure 22 this is indeed the case, except for the gentle case I.

\section{The dam-break problem}

In the dam-break problem a mound of water on a flat bed is released from rest under action of gravity. Most attention has been given to the case with a semi infinite fluid shelf with a vertical front. At the first instant a potential flow solution of the problem, yielding pressures and accelerations, is readily obtained by Fourier series. At the toe of the fluid front the potential solution displays a singularity giving infinite values for the acceleration. The presence of this feature is easily inferred from the structure of the boundary value problem. At the free surface the pressure is zero, while the normal derivative at the bottom must counterbalance the gravity to give zero vertical acceleration. At a contact point between the free surface and the bottom this implies large horizontal gradients for contact angles close to $90^{\circ}$, whereas perpendicular contact leads to a double valued vertical derivative and singularity. For large times there exist asymptotic shallow water solutions for the evolution of the fluid front. The key point is that the front rapidly evolves into a thin tongue with no bore like features. This kind of dam-break problem is reviewed in Stoker (1957), while a fresh experimental and theoretical investigation is reported in Stansby et al. (1998). Also other initial shapes have been investigated, with relevance to, for instance, the collapse of fluid columns produced by underwater explosions.

A simple initial shape, not very different from the wave front in the early stages of run-up, is that of a semi circle. Again discrete Fourier series can be applied for the 


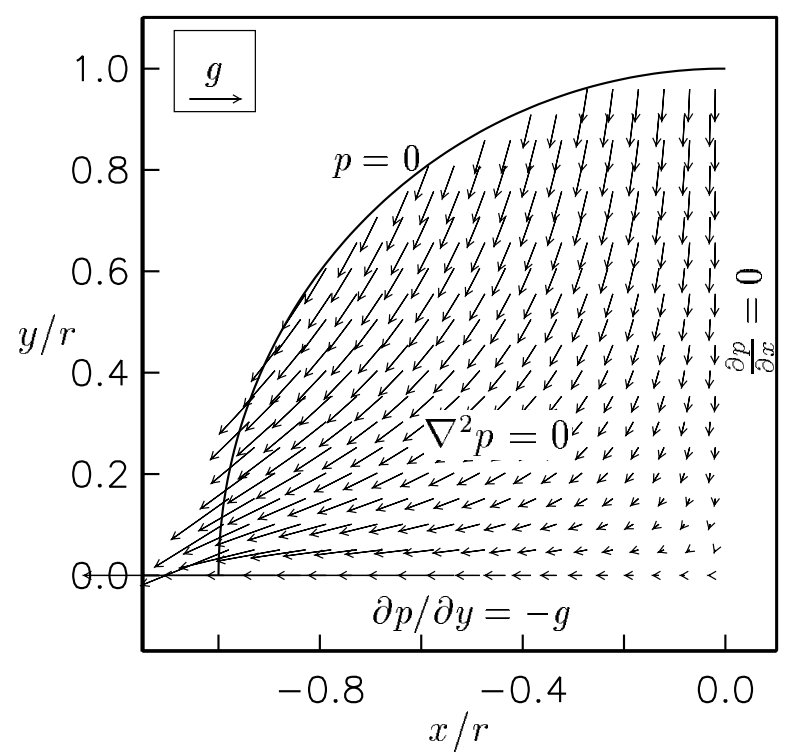

Figure 23: Accelerations from the "circular" dam-break problem. The size of the reference arrow corrosponds to the constant of gravity. The definition of the dambreak boundary value problem, formulated in terms of the pressure, is also indicated in the figure.

initial pressure distribution. Details are given in Martin et al. (1952), that also contains numerical solutions for larger times, still based on Fourier series. For the acceleration at the free surface Martin et al. (1952) obtained the closed form expression

$$
a_{r}=-\frac{2 g}{\pi}\left(1+\cos \theta \ln \left(\tan \frac{\theta}{2}\right)\right),
$$

where $\theta$ is the angle between the radii and the positive horizontal axis. We note that $a_{r}$ is scaled only by $g$ and that the tangential acceleration equals the tangential component of gravity. The acceleration distribution in the interior is given by a Fourier series that converges except at the contact points. The result is displayed in figure 23. In spite of the differences with respect to surface shape etc. the similarity with the measured accelerations in figure 17 is striking.

Naturally, the Boussinesq equations are not likely to reproduce the circular dambreak problem. However, in view of the relations between this problems and run-up, it would be instructive to observe how long wave theory fails. In fact, generalized dambreak problems, including cases with contact angles less than $90^{\circ}$ would probably serve excellent as benchmark problems for the applicability of simplified theories in general. Assuming horizontal bottom and zero velocities the Boussinesq equations (8,9) simplify and we may readily obtain second order ordinary differential equation for either $\frac{\mathrm{D} \bar{u}}{\mathrm{~d} t}$ or $\frac{\mathrm{D}^{2} H}{\mathrm{~d} t^{2}}$ at $t=0$

$$
\begin{aligned}
& \frac{\mathrm{D} \bar{u}}{\mathrm{~d} t}-\frac{\beta}{3 H} \frac{\mathrm{d}}{\mathrm{d} x}\left(H^{3} \frac{\mathrm{d}}{\mathrm{d} x} \frac{\mathrm{D} \bar{u}}{\mathrm{~d} t}\right)=-\frac{\mathrm{d} H}{\mathrm{~d} x}, \\
& \frac{\mathrm{D}^{2} H}{\mathrm{~d} t^{2}}-\frac{\beta}{3 H} \frac{\mathrm{d}}{\mathrm{d} x}\left[\frac{1}{H} \frac{\mathrm{d}}{\mathrm{d} x}\left(H^{2} \frac{\mathrm{D}^{2} H}{\mathrm{~d} t^{2}}\right)\right]=H \frac{\mathrm{d}^{2} H}{\mathrm{~d} x^{2}},
\end{aligned}
$$

where the scaling is as in appendix B. We observe that the left hand operators becomes regularly singular when $H$ has a zero of order 1 or $\frac{1}{2}$. When the contact angle is zero, 

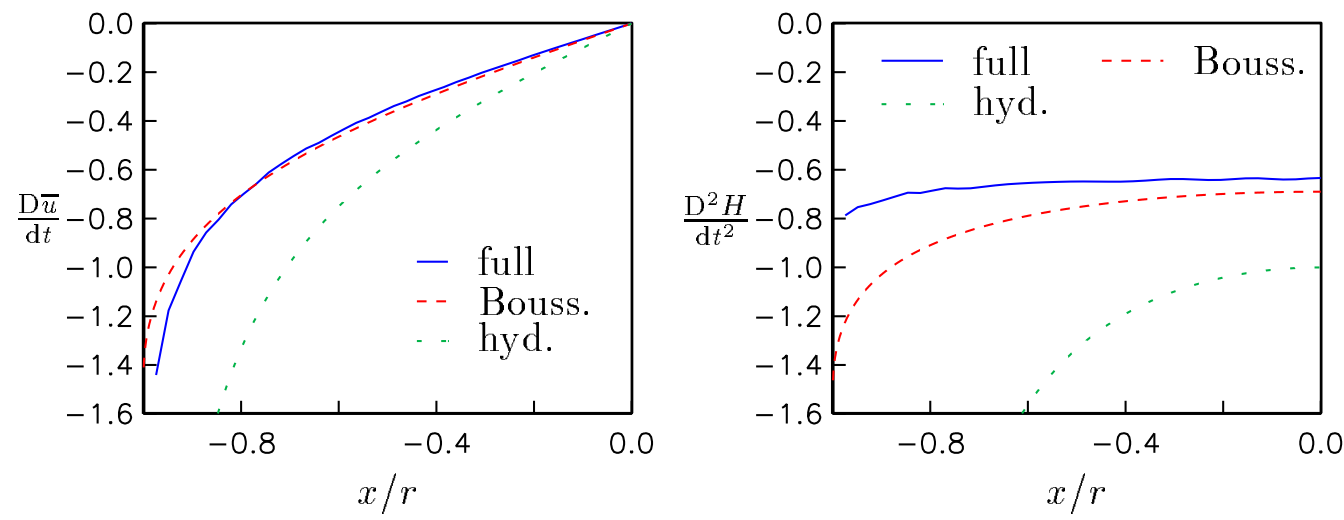

Figure 24: Comparison of full potential theory and long wave equations for the circular dambreak problem. The accelerations are scaled by gravity.

as for a semi circle, also the right hand sides are singular. In the hydrostatic case this singularity is conveyed directly to the solutions and we obtain singularities that are much stronger than for full potential theory. When the dispersive terms are retained, on the other hand, the singularity vanish and the accelerations becomes finite at the contact points ${ }^{1}$. Even though the absence of a singularity is in accordance with the physics, it must be regarded as a shortcoming of the Boussinesq theory. For the run up simulations the boundary conditions at the fluid tip was $H=0$. This is not consistent with the behavior of $\frac{\mathrm{D}^{2} H}{\mathrm{~d} t^{2}}$ as given by (16). However, in numerical solutions of the equations we obtain convergence, when refining the grid, toward a unique solution, regardless of the boundary condition mismatch. The solutions are displayed in figure 24 together with the corresponding quantities from the full potential flow solution. Apart from the neighborhood of the contact point the Boussinesq solution displays very good accuracy for $\frac{\mathrm{D} \bar{u}}{\mathrm{~d} t}$. The agreement for $\frac{\mathrm{D}^{2} H}{\mathrm{~d} t^{2}}$ is not quite as good, indicating that Boussinesq theory is challenged not only by the steep gradients at the contact point, but also by the small overall length to depth ratio of the fluid body. Still the Boussinesq solution is much better than the hydrostatic one and they both predict large horizontal accelerations at the fluid tip.

\footnotetext{
${ }^{1}$ We have local expansions like $\frac{\mathrm{D} \bar{u}}{\mathrm{~d} t}=\gamma+\kappa\left(x-x_{c}\right)^{\frac{1}{2}}+.$. , where $x_{c}$ is the position of the contact point.
} 


\section{References}

Carrier, G. F. \& Greenspan, H. P. 1958 Water waves of finite amplitude on a sloping beach. J. Fluid Mech. 4, 97-109.

Chang, K.-A. \& LiU, P. L.-F. 1998 Velocity, acceleration and vorticity under a breaking wave. Physics of fluids 10, 327-329.

Chang, K.-A. \& LiU, P. L.-F. 1999 Experimental investigation of turbulence generated by breaking waves in water of intermediate depth. Physics of fluids 11, 33903400 .

Craig, K. R. \& Thieke, R. J. 1996 Application of a digital particle image velocimetry (dpiv) system to breaking waves in the surf zone. In 25th International conference on Coastal Engineering (ed. B. Edge), pp. 602 - 615. ASCE.

DABIRI, D. \& GhARIB, M. 2001 Simultaneous free-surface deformation and nearsurface velocity measurements. Experiments in Fluids 30, 381-390.

Flather, R. A. \& Heaps, N. S. 1975 Tidal computations for morecambe bay. Geophys. J. R. Astron. Soc. 42, 489-517.

Grilli, S. \& Svendsen, I. A. 1990 Computation of nonlinear wave kinematics during propagation and run-up on a slope, pp. 378-412. Kluwer Academic Publishers.

Hibberd, S. \& Peregrine, D. H. 1979 Surf and run-up on a beach: a uniform bore. J. Fluid Mech 95, 323-345.

Jakobsen, M. L., Dewhirst, T. P. \& Greated, C. A. 1997 Particle image velocimetry for predictions of acceleration fields and force within fluid flows. Meas. Sci. Technol. 8, 1502-1516.

Jensen, A., Sveen, J. K., Grue, J., Richon, J.-B. \& Gray, C. 2001 Accelerations in water waves by extended particle image velocimetry. Experiments in Fluids 30, $500-510$.

Johnsgard, H. 1999 A numerical model for run-up of breaking waves. Int. J. Num. Meth. Fluids 31, 1321-1331.

Johnsgard, H. \& Pedersen, G. 1997 A numerical model for three-dimensional run-up. Int. J. Num. Meth. Fluids 24, 913-931.

Keller, H. B., Levine, A. D. \& Whitham, G. B. 1960 Motion of a bore over a sloping beach. J. Fluid Mech. 7, 302-316.

Keulegan, G. H. 1948 Gradual damping of solitary waves. J. Res. Natl. Bur. Stand. 40, 497-498.

Kowalik, Z. \& Murty, T. S. 1993 Numerical simulation of two-dimensional tsunami run-up. Marine Geodesy 16, 87-100. 
Lin, P., Chang, K.-A. \& LiU, P. L.-F. 1999 Runup and rundown of solitary waves on sloping beaches. Journal of Waterway, Port, Coastal and Ocean Engineering 125 (5), 247-255.

Lin, P. \& LIU, P. L.-F. 1998 a A numerical study of breaking waves in the surf zone. Journal of Fluid Mechanics 359, 239-264.

Lin, P. \& LiU, P. L.-F. $1998 b$ Turbulence transport,vorticity dynamics, and solute mixing under plunging breaking waves in surf zone. Journal of Geophysical Research 103, $15677-15694$.

Martin, J. C., Moyce, W. J., Penney, W. G., Price, A. T. \& Thornhill, C. K. 1952 Some gravity wave problems in the motion of perfect liquids. Philosofical Transactions of Royal Society of London, Ser. A 244, 231-334.

Meyer, R. E. \& Taylor, A. D. 1972 Run-up on beaches. In Waves on Beaches and Resulting Sediment Transport (ed. R. E. Meyer), pp. 357-411. Academic.

Nadoaka, K., Hino, M. \& Koyano, Y. 1989 Structure of the turbulent flow field under breaking waves in the surf zone. Journal of Fluid Mechanics 204, 359-387.

New, A. L., McIver, P. \& Peregrine, D. H. 1985 Computations of overturning waves. Journal of Fluid Mechanics 150, 233-251.

Pedersen, G. \& Gjevik, B. 1983 Run-up of solitary waves. J. Fluid Mech. 135, $283-299$.

Peregrine, D. H. \& Williams, S. M. 2001 Swash overtopping a truncated plane beach. Accepted for publication in Journal of Fluid Mechanics.

Raffel, M., Willert, C. E. \& Kompenhans, J. 1998 Particle Image Velocimetry, A Practical Guide, 1st edn. Springer Verlag.

Shen, M. C. \& Meyer, R. E. 1963 Climb of a bore on a beach, 3:run-up. J. Fluid Mech. 16, 113-125.

Shuto, N. 1976 Transformation of nonlinear long waves. Coastal engineering pp. 423440.

Stansby, P. K., Chegini, A. \& Barnes, T. C. D. 1998 The initial stages of dam-break flow. Journal of Fluid Mechanics 374, 407-424.

STOKER, J. 1957 Water waves. Interscience.

SynOLAKIS, C. E. 1987 The run-up of solitary waves. J. Fluid Mech. 185, 523-545.

Tanaka, M. 1986 The stability of solitary waves. Phys. Fluids 29(3), 650-655.

Ting, F. C. K. \& Kirby, J. T. 1995 Dynamics of surf-zone turbulence in a strong plunging breaker. Coastal Engineering 24, 177-204.

Ting, F. C. K. \& Kirby, J. T. 1996 Dynamics of surf-zone turbulence in a spilling breaker. Coastal Engineering 27, 131-160. 
Zelt, J. \& Raichlen, F. 1990 A Lagrangian model for wave-induced harbour oscillations. J. Fluid Mech. 213, 203-225. 